\title{
Two-fluid solutions for Langmuir probes in collisionless and isothermal plasma, over all space and bias potential
}

Ph. Guittienne, ${ }^{1}$ A. A. Howling, ${ }^{2}$ and I. Furno ${ }^{2}$

${ }^{1)}$ Helyssen, Route de la Louche 31, CH-1092 Belmont-sur-Lausanne, Switzerland

${ }^{2)}$ Ecole Polytechnique Fédérale de Lausanne (EPFL), Swiss Plasma Center, CH-1015 Lausanne, Switzerland

(Dated: 5 September 2018)

This paper presents solutions for the classical one-dimensional (radial and Cartesian) problem of Langmuir probes in a collisionless, isothermal plasma. The method is based on two-fluid equations derived from the first two moments of Vlasov's equation. In contrast to commonly-used approximations, electron inertia and ion temperature are not neglected so that the fluid equations are symmetric in the terms for electrons and ions. The fluid equations are reduced analytically so that the electric potential is the only remaining spatial function, which is numerically determined using Poisson's equation. The single radial solution applies continuously over the whole region from the probe up to the unperturbed plasma, in contrast to theories which separate the probe boundary region into a charged sheath and a quasi-neutral pre-sheath, and is valid for all values of probe bias potential. Current-voltage characteristics are computed for cylindrical and spherical probes, which exhibit non-saturation of the ion and electron currents. The 1D Cartesian case is also analysed, and the Bohm criterion is recovered only in the limit of large radius probes.

\section{INTRODUCTION}

A body immersed in a plasma acts as a sink for charged particles. Because of the large difference in mobility between electrons and ions, the former tend to be lost more rapidly to the body, thus creating a charged region in the vicinity of the surface, commonly called the sheath. When the immersed object is electrically floating, the space charge distribution in the sheath evolves towards an equilibrium where ion and electron fluxes are equal and no more charge is transferred to the body. If the immersed object is biased, the sheath adapts to the imposed boundary potential and a current is collected.

A fundamental diagnostic in plasma physics, the Langmuir probe, relies on the analysis of the current-voltage characteristic obtained by sweeping the bias potential on an immersed metallic pin. The method is technically simple, but the analysis of Langmuir probe characteristics is difficult, mainly because of the theoretical issues inherent to the plasma sheath problem. ${ }^{1}$ This was notably addressed by Langmuir and colleagues ${ }^{2,3}$ and has since been a major topic in plasma physics. A fundamental set of equations involved in this problem arises from the moments of Boltzmann's equation which describe the electrons and ions as two fluids in terms of their mean densities and velocities. The successive moments by themselves do not constitute a closed set of equations and have to be completed by a closure expression. Furthermore, the two fluid equations are coupled through Poisson's equation which determines the electric potential.

In the early stages of sheath theory, ${ }^{4}$ Langmuir introduced two approximations which have been almost universally adopted up till now: The first is to separate the continuous region next to the probe into a spacecharge sheath and a quasi-neutral plasma $;^{1-18}$ the interpretation of the plasma-sheath transition has caused much debate. ${ }^{1,4,15,16}$ The second approximation is to neglect the electron inertia in the associated momentum conservation equation, which results in the commonlyused Boltzmann relation for the electron density, ${ }^{1-28}$ $n_{e}=n_{0} \exp \frac{\Delta V}{T_{e}}$, where $\Delta V$ and $T_{e}$ are respectively a potential difference and the electron temperature, here expressed in volts. ${ }^{28}$

In this work, we present two-fluid solutions of a collisionless, isothermal plasma with singly-charged positive ions, for the simplified situations of one-dimensional (1D) radial and Cartesian geometries. To be precise, the problem is solved analytically until the point where Poisson's equation has to be integrated, which is performed numerically. Specific features of the solution method are the retainment of electron inertia and ion temperature in the fluid momentum equations, the use of Lambert's function, ${ }^{29-31}$ and the respect of particle flux conservation everywhere from the unperturbed plasma up to the probe surface. Boundary conditions are of prime importance because they define the probe fluxes; these are calculated from the self-consistent convective flow velocity of each species.

As a first consequence, the separation of space into sheath and quasi-neutral plasma regions is no longer necessary for radial probes because a single, collisionless solution is considered. Secondly, the fluid equations are symmetric in the terms for electrons and ions (accounting for charge sign), hence the solution is valid for all probe bias voltages. Current-voltage characteristics can be derived analytically for the 1D Cartesian case, whereas for cylindrical and spherical probes, two parameters still have to be determined numerically. 


\section{A. Brief review of probe theory}

The dominant approach to describe the perturbed region adjacent to a plane probe consists in splitting this region into two distinct ones. The first sub-region, the sheath, extends from the probe surface to a point, the sheath entrance, at which the electron and ion densities are taken to be equal. Beyond this point there extends a second sub-region, the pre-sheath, characterized by the quasi-neutrality hypothesis. Collisionless and isothermal 1D Cartesian models are generally applied to describe the sheath region, which is then characterized by constant particle fluxes. The Bohm criterion ${ }^{5}$ was developed to account for the ion velocity at the sheath entrance. Ionization must then be considered to provide the necessary ion flux in the pre-sheath. This division between sheath and pre-sheath regions gives rise to 2-scale theories. ${ }^{3,5,6,11,12}$ Different assumptions and approximations are made in these domains, for example, collisional ionisation in the pre-sheath and no collisions in the sheath, ${ }^{6}$ so it is clear that matching problems will occur at the sheath/pre-sheath interface. ${ }^{7,12}$ This can be partly redressed by interposing another domain, a transition layer, on an intermediate scale, which accounts for space charge and the dominant pre-sheath processes. ${ }^{12}$ In this way, as more layers are added, the approximations are successively improved for the various scale lengths. It is clear, however, that a single analytical solution for plasma-to-wall is precluded, by definition. ${ }^{7}$ Ideally, the exact Boltzmann equation would be solved over the whole region to give a single solution. In the absence of such analytical solutions, single profiles were obtained by numerical computations which do not require multi-scale assumptions ${ }^{7,32-35}$ although their accuracy of course depends on any approximations made. Furthermore, real finite-sized plane probes collect ions from an ill-defined hemispherical shape so that, in practice, an infinite plane wall plasma-sheath transition does not exist ${ }^{1,20}$ and at least a 2D approach would be necessary. ${ }^{31}$

To avoid the difficulties associated with infinite plane walls, this paper goes on to consider a two-fluid, 1D solution for radial Langmuir probes (cylindrical and spherical) in collisionless, isothermal plasma. The term "fluid" conventionally refers to collisional conditions; however, the macroscopic variables for electrons and ions are here described by velocity moments of two collisionless distribution functions, often known as a two-fluid approach. In the case of radial probes, the ion acceleration due to inward flux concentration in the "geometric pre-sheath" means that a collisionless pre-sheath solution relaxes asymptotically to the unperturbed, field-free plasma at infinity, without requiring ionisation or other collisional processes which are necessary in the $1 \mathrm{D}$ plane wall situation. ${ }^{11,12}$ Hence a collisionless, single, 1D radial solution would appear possible for both the sheath and the pre-sheath, i.e. over the whole domain. ${ }^{1}$ In fact, the applicability, ${ }^{18}$ or necessity, ${ }^{1,7,20,35}$ of the Bohm criterion is less clear for cylindrical and spherical probe ge- ometries. Calculations in the limit of probe radius much larger than the sheath width ${ }^{36}$ are used here to reconcile the $1 \mathrm{D}$ radial model with conventional $1 \mathrm{D}$ sheath/presheath models ${ }^{11,12}$ which invoke the Bohm criterion.

Fluid models for non-saturated probe ion currents calculate the increase of the effective ion collection area due to a thicker sheath at higher values of probe bias voltage. Non-saturated currents are also predicted in the framework of kinetic approaches, notably in orbitalmotion-limited (OML) theories. ${ }^{2,8,19,21,27}$ In these, the conservation of individual particle angular momentum plays a central role. In the present work, we develop a two-fluid solution under the hypothesis of isotropic temperatures and Maxwellian distributions. These assumptions are common, ${ }^{11,25,30-32}$ and fair agreement is found with a kinetic model. ${ }^{37}$ However, it means that any distortions of the Maxwellian distributions due to truncation, ${ }^{7,27,31,38,39}$ anisotropic temperatures, ${ }^{37}$ angular momentum conservation or trapped orbits ${ }^{21,32}$ cannot be accounted for. This does not mean that kinetic effects are unimportant; in fact, orbital motion kinetic effects are expected to be more and more relevant as the probe diameter becomes smaller, making the assumption of Maxwellian distributions close to the probe surface less valid. This is probably a limit of validity of the present Maxwellian approach. To have a better twofluid collisionless description, one should calculate the distortions of the distribution functions due to kinetic effects (such as in OML theory) before performing Vlasov equation moments. Instead, this two-fluid model tacitly assumes that like-particle collisions are sufficiently frequent to maintain Maxwellian distributions, even near to an adsorbing wall, but that unlike-particle collisions are sufficiently rare for friction and ionisation to be negligible. Alternatively, there may be other processes, such as turbulence or electric field micro-instabilities, which maintain a Maxwellian distribution even when collisions are rare; this is known as Langmuir's paradox. ${ }^{25}$ Finally, fluctuations and instabilities such as ion acoustic waves could affect experimental measurements but are not considered in the stationary solutions presented here. ${ }^{31,40}$ To summarize, this fluid model might not represent physical situations generally, but it is a solution to a classical problem of probes in collisionless, isothermal plasma.

\section{B. Structure of the paper}

The analytical model for a probe in a collisionless plasma is developed in three stages corresponding to the three geometries in Fig. 1:

1. Fig. 1(a) shows a 1D Cartesian model for collisionless plasma flow to an infinite plane wall. The wall does not represent a non-intrusive Langmuir probe causing only a small perturbation to the plasma. ${ }^{1,31}$ Nevertheless, this 1D Cartesian model serves the purpose of introducing the mathematical method in 

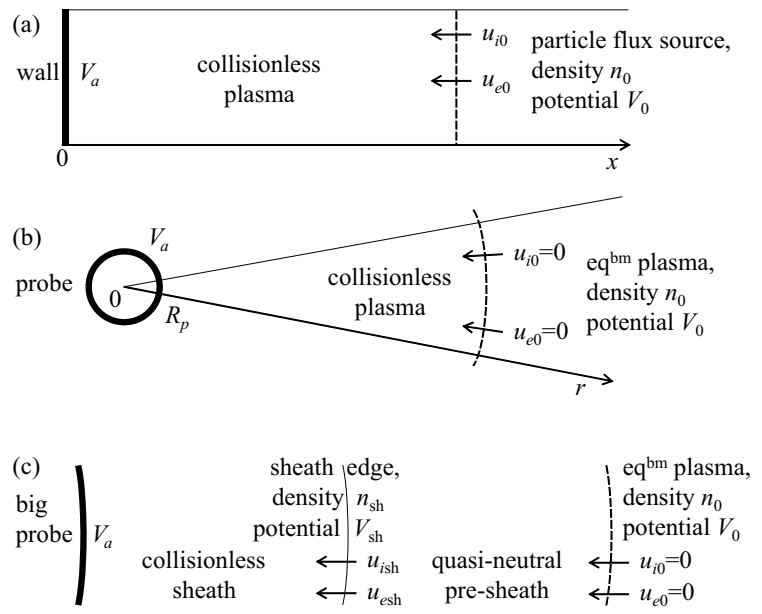

FIG. 1. Basic description of the probe geometries, showing the probe applied voltage $V_{a}$, and the ion and electron convective velocities $u_{i 0}$ and $u_{e 0}$. The dashed lines represent the limit of large distance from the probe up to the equilibrium plasma at infinity. (a) Infinite plane wall (1D Cartesian solution) in Section II, with incoming particle velocities from a supposed plasma source at infinity. ${ }^{41}$ (b) Cylindrical and spherical probes (1D radial solution) of Sections III and IV, with vanishing particle velocity from infinity. (c) Big radius probe in Section V, with space charge sheath and quasineutral pre-sheath, showing the ion and electron convective velocities $u_{i s h}$ and $u_{e s h}$ at the plasma-sheath interface.

Section II, and provides a comparison in the limit of large-radius probes in Section V.

2. Fig. 1(b) shows a 1D radial model to represent collisionless plasma flow to a probe. In contrast to the 1D Cartesian model, the flux to a small radius Langmuir probe causes only a small perturbation to the plasma, which is maintained by some independent source. Almost all of the ionisation rate due to this independent source is assumed to be in equilibrium with particle loss rates to other electrodes and surfaces. The assumption of collisionless plasma in the locality of the probe remains a reasonable approximation for a perturbed region around the probe which is small compared to the mean free path for particle collisions. The length-scale of the solution then determines the lower limit of the mean free path and hence the upper pressure limit for solution validity. ${ }^{34}$ For perturbation scale lengths $<1 \mathrm{~mm}$ calculated in this work, collisionless conditions are expected to be satisfied for argon gas pressures up to about $1 \mathrm{~Pa}$, where the mean free path for collisions $\lambda_{c} \sim 1 \mathrm{~cm}$ (for ion charge-exchange collision cross-sections $^{28,34} \sim 4 \cdot 10^{-19} \mathrm{~m}^{2}$ ). Cylindrical and spherical probe $1 \mathrm{D}$ radial solutions are presented in Sections III and IV respectively.

3. Fig. 1(c) shows part of the wall of a big radius probe in a 1D radial model. Section V compares the limit of large probe radius with the 1D Cartesian plane wall solution and a classical sheath model.

Discussions and conclusions are presented in Sections VI and VII respectively. Finally, Appendixes A and B explain the Lambert solution and describe a practical method to interpret probe $I V$ characteristics.

\section{1D CARTESIAN CASE OF AN INFINITE PLANE WALL}

\section{A. Conservation of particles and of momentum for electrons and ions}

Taking the first velocity moment of Vlasov's equation, a momentum conservation equation for ions and electrons can be written for a two-fluid description as follows:

$$
m_{s} n_{s}\left[\frac{\partial \mathbf{u}_{s}}{\partial t}+\left(\mathbf{u}_{s} \cdot \nabla\right) \mathbf{u}_{s}\right]=q_{s} n_{s} \mathbf{E}-\nabla \cdot \mathbf{P}_{s}
$$

for a collisionless, non-magnetized plasma, where $n_{s}$ is the number density of species $s, \mathbf{u}_{s}$ the convective velocity, $m_{s}$ the particle mass, $q_{s}$ the electric charge (sign included), $\mathbf{P}_{s}$ the pressure tensor, and $\mathbf{E}$ the electric field.

We consider here the 1D Cartesian problem $\left(\nabla \rightarrow \partial_{x}\right)$ of a semi-infinite plasma facing a wall with steady state flow $\left(\partial_{t} \rightarrow 0\right)$ in Fig. 1(a). The solutions here are restricted to the case of electrons and a single ion species with a single positive charge $\left(\left|q_{s}\right|=q\right.$, the elementary charge). The treatment of different ion species, multiplycharged ions, and negative ions, although possible, is beyond the scope of this work. As a closure expression for (1) we assume an isothermal relation for equilibrium, with isotropic Maxwellian velocity distributions for each species. ${ }^{25,31,40}$ Under these conditions the pressure tensor is diagonal and isotropic, and by using the ideal gas law the scalar pressure can be expressed as $p_{s}=n_{s} q T_{s}$. Here, $T_{s}$ is the $s$ species mean temperature defined in volts in order to simplify expressions involving ratios of voltage and temperature. ${ }^{28}$ The relation between $T_{s}$ in volts, and the temperature $\mathrm{T}_{s}$ in Kelvin, is given by $q T_{s}=k_{\mathrm{B}} \mathrm{T}_{s}$, where $k_{\mathrm{B}}$ is Boltzmann's constant, by which the conventional expressions can be recognised.

The resulting fluid equations for electron and ion momentum are:

$$
\begin{array}{r}
m_{e} n_{e} u_{e} \partial_{x} u_{e}-q n_{e} \partial_{x} V+q T_{e} \partial_{x} n_{e}=0, \\
m_{i} n_{i} u_{i} \partial_{x} u_{i}+q n_{i} \partial_{x} V+q T_{i} \partial_{x} n_{i}=0,
\end{array}
$$

where $V$ is the electric potential. Here, the densities, fluid velocities, and potential are all functions of $x$.

In the literature, at this point, the electron inertia convective term (proportional to $m_{e}$ ) is almost universally neglected in (2), and as such, the electron density is described by a Boltzmann relation. ${ }^{1-28}$ Furthermore, the cold ion approximation is usually applied $\left(T_{i}=0\right)$ in which case the ion pressure gradient is neglected and (2) 
leads to the free fall solution for the ion density. A consequence of these approximations is that the sheaths are only properly described for large negative bias potentials with respect to the plasma potential. In the following, we break with tradition and keep all terms in (2). In fact, the only way to guarantee validity for the whole range of probe bias potential is to maintain symmetry between the electron and ion terms. ${ }^{32}$ It will be seen that the electron and ion variables are interchangeable in all the exact expressions - accounting for charge sign - as is necessary for a model where the probe bias potential can be lower or higher than the plasma potential.

We assume $n_{e}, n_{i} \neq 0$ for all $x$, and define thermal velocities $u_{\text {the }}=\sqrt{q T_{e} / m_{e}}$ and $u_{\text {th } i}=\sqrt{q T_{i} / m_{i}}$, the root mean square velocity in any single direction. The mathematical expressions can be simplified by introducing the dimensionless functions $U_{e}, U_{i}$ which are the squares of the convective velocity normalized to the thermal velocity, for electrons and ions respectively:

$$
U_{e}=\left(\frac{u_{e}}{u_{\mathrm{the}}}\right)^{2} \text { and } \quad U_{i}=\left(\frac{u_{i}}{u_{\mathrm{th} i}}\right)^{2} .
$$

Equations (2) then become:

$$
\begin{aligned}
\frac{1}{2} \partial_{x} U_{e}-\frac{1}{T_{e}} \partial_{x} V+\partial_{x} \ln \left(n_{e}\right) & =0, \\
\frac{1}{2} \partial_{x} U_{i}+\frac{1}{T_{i}} \partial_{x} V+\partial_{x} \ln \left(n_{i}\right) & =0 .
\end{aligned}
$$

Neglecting ionisation and recombination processes, consistent with the collisionless assumption, the particle flux conservation is expressed from the zeroth moment of Vlasov's equation as:

$$
\partial_{t} n_{s}+\nabla \cdot\left(n_{s} \mathbf{u}_{s}\right)=0,
$$

which, according to our assumptions, leads to:

$$
\partial_{x}\left(n_{e} u_{e}\right)=0, \quad \text { and } \quad \partial_{x}\left(n_{i} u_{i}\right)=0 .
$$

Equations (3) and (5) can be directly integrated to give:

$$
\begin{array}{r}
\frac{U_{e}}{2}-\frac{V}{T_{e}}+\ln \left(n_{e}\right)=C_{e}, \\
\frac{U_{i}}{2}+\frac{V}{T_{i}}+\ln \left(n_{i}\right)=C_{i}, \\
n_{e} u_{e}=\phi_{e}, \quad \text { and } n_{i} u_{i}=\phi_{i},
\end{array}
$$

where the integration constants $C_{e}, C_{i}, \phi_{e}, \phi_{i}$ depend on the boundary conditions. $\phi_{e}, \phi_{i}$ are the constant flux densities (particles per second per unit area) for electrons and ions respectively. Electrons and ions are assumed to disappear by neutralization at the wall, with no processes such as secondary emission. For the plasma at infinity, we set an equal density $n_{0}$ for electrons and ions, and the plasma potential is set to $V_{0}$. Because we aim to treat the artificial 1D Cartesian problem of a semi-infinite collisionless plasma facing a plane wall, we must, considering (7), set non-vanishing injection velocities $u_{s 0}$ from infinity to ensure the conservation of particle fluxes, as shown

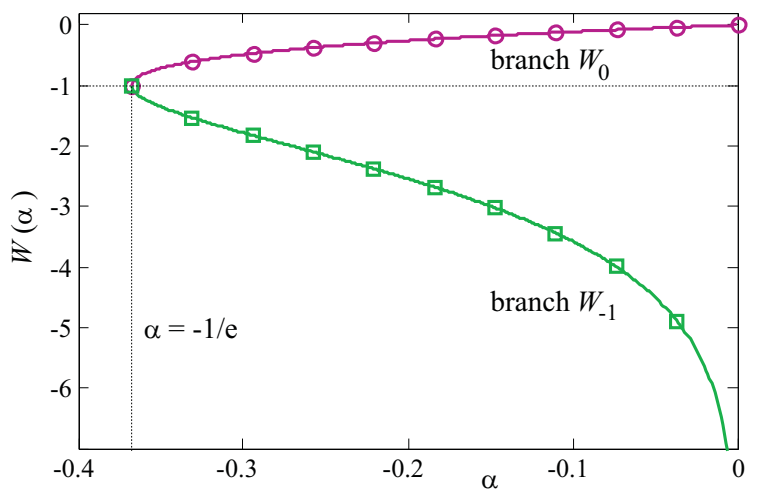

FIG. 2. Plot of the Lambert function branches $W_{0}$ (circles) and $W_{-1}$ (squares) which have purely real and negative values for $-\frac{1}{\mathrm{e}} \leq \alpha \leq 0$.

in Fig. 1(a). We therefore define the non-vanishing values at infinity for the normalized square velocities to be $U_{s 0}=\left(u_{s 0} / u_{\mathrm{ths}}\right)^{2}$. Applying these boundary conditions to (6) and (7), the integration constants $C_{e}, C_{i}, \phi_{e}$ and $\phi_{i}$ are determined so that we can express $n_{e}$ and $n_{i}$ as:

$$
\begin{aligned}
& n_{e}=n_{0} \exp \left(\frac{U_{e 0}-U_{e}}{2}+\frac{V-V_{0}}{T_{e}}\right), \\
& n_{i}=n_{0} \exp \left(\frac{U_{i 0}-U_{i}}{2}-\frac{V-V_{0}}{T_{i}}\right),
\end{aligned}
$$

and the particle flux conservation (7) as:

$$
n_{e}^{2} U_{e}=n_{0}^{2} U_{e 0}, \quad \text { and } \quad n_{i}^{2} U_{i}=n_{0}^{2} U_{i 0} .
$$

The particle density equations (8) are a more general form of the Boltzmann relation, known as the "Boltzmann distribution law" in kinetic theory, where the density, fluid velocity and potential can be non-uniform. ${ }^{42}$ Combining these relations to eliminate the particle densities gives expressions for the particle fluid velocities in terms of the potential:

$$
\begin{gathered}
-U_{e} \mathrm{e}^{-U_{e}}=-U_{e 0} \mathrm{e}^{-U_{e 0}-\frac{2\left(V-V_{0}\right)}{T_{e}}}, \\
-U_{i} \mathrm{e}^{-U_{i}}=-U_{i 0} \mathrm{e}^{-U_{i 0}+\frac{2\left(V-V_{0}\right)}{T_{i}}} .
\end{gathered}
$$

The transcendental equations (9) have for solutions:

$$
\begin{gathered}
U_{e}=-W\left[-U_{e 0} \mathrm{e}^{\left.-U_{e 0}-\frac{2\left(V-V_{0}\right)}{T_{e}}\right]}=-W\left[\alpha_{e}\right],\right. \\
U_{i}=-W\left[-U_{i 0} \mathrm{e}^{-U_{i 0}+\frac{2\left(V-V_{0}\right)}{T_{i}}}\right]=-W\left[\alpha_{i}\right],
\end{gathered}
$$

where $W$ denotes the Lambert function ${ }^{29}$ defined by $\alpha=z \mathrm{e}^{z} \Leftrightarrow z=W[\alpha]$. This gives the functional dependence of the fluid velocities and densities on the electric potential, which is then the only remaining function to be determined using Poisson's equation. In the following we will first analyse the consequences of solutions (10); the full solution requiring integration of Poisson's equation is carried out in Sec. IID. 
The Lambert function, which cannot be expressed in terms of elementary functions, is multi-valued and therefore has several branches. Because $U_{e}$ and $U_{i}$ are square velocities, the solutions (10) must be real and positive, which implies that the Lambert functions must be real and negative. Fortunately, regarding the former condition, only two branches of the Lambert function, $W_{0}$ and $W_{-1}$, have purely real values. Furthermore, in order to obtain negative values, the arguments for $W_{0}$ and $W_{-1}$ must be restricted to the interval $\left[-\frac{1}{\mathrm{e}}, 0\right]$. The representations of branches $W_{0}(\alpha)$ and $W_{-1}(\alpha)$ in this interval are shown in Fig. 2. The branch point, $\alpha=-\frac{1}{\mathrm{e}}$, is of particular interest as it is the only value for which the condition $W_{0}(\alpha)=W_{-1}(\alpha)$ can be fulfilled. Note that, as $W_{0,-1}\left(-\frac{1}{\mathrm{e}}\right)=-1$, the condition $\alpha_{s}=-\frac{1}{\mathrm{e}}$ is equivalent to $u_{s}=u_{\text {ths }}$. Then, considering a given $s$ species, the $W_{0}$ branch of the Lambert function must be applied to describe the fluid velocity $u_{s}$ when it is known to be less than, or equal to, the thermal velocity. On the contrary, if $u_{s} \geq u_{\mathrm{th} s}$, the fluid velocity must be described by the $W_{-1}$ branch. Using these properties of the Lambert function, and under the important hypothesis of a monotonic spatial variation of the electric potential $V(x)$, from infinity where $V(x)=V_{0}$ up to the wall surface where a bias potential $V_{a}$ is applied, the analysis of conditions $\alpha_{e, i} \in\left[-\frac{1}{\mathrm{e}}, 0\right]$ (see Appendix A) leads to the following conclusions:

The applied potential $V_{a}=V_{0}$ plays a particular role in this problem because it corresponds to a field free situation. For $V_{a}<V_{0}$, ions are the attracted species and electrons are repelled, while the opposite happens when $V_{a}>V_{0}$. The roles of ions and electrons are then, in a way, symmetric with regard to the plasma potential.

$$
\begin{aligned}
& \text { For } V_{a} \leq V_{0}: \\
& \qquad U_{e}=-W_{0}\left[\alpha_{e}\right] \text { and } U_{i}=-W_{-1}\left[\alpha_{i}\right],
\end{aligned}
$$

with the conditions on initial velocities:

$$
\begin{aligned}
& U_{i 0} \geq 1 \\
& 0 \leq U_{e 0} \leq U_{e 0}^{\max }=-W_{0}\left[-\mathrm{e}^{\frac{2\left(V_{a}-V_{0}\right)}{T_{e}}-1}\right] \leq 1
\end{aligned}
$$

The relations (12) imply that ions come from infinity with an initial convective velocity $u_{i 0}$ greater or equal to $u_{\mathrm{th} i}$. The initial velocity of electrons, $u_{e 0}$, is limited to a maximum value $u_{e 0}^{\max }$. For $u_{e 0}=u_{e 0}^{\max }$, the electron velocity at the wall is also maximum and is equal to the thermal velocity $u_{\text {the }}$.

$$
\begin{aligned}
& \text { For } V_{a} \geq V_{0} \text { : } \\
& \qquad U_{e}=-W_{-1}\left[\alpha_{e}\right] \text { and } U_{i}=-W_{0}\left[\alpha_{i}\right],
\end{aligned}
$$

with the conditions on initial velocities:

$$
\begin{aligned}
U_{e 0} & \geq 1 \\
0 \leq U_{i 0} \leq U_{i 0}^{\max }=-W_{0}\left[-\mathrm{e}^{-\frac{2\left(V_{a}-V_{0}\right)}{T_{i}}-1}\right] & \leq 1 .
\end{aligned}
$$

As stated above, the roles of electrons and ions are inverted when $V_{a}$ crosses the plasma potential value, so that electrons now come from infinity with an initial convective velocity higher than their thermal velocity, while the maximal ion velocity at the wall is $u_{\mathrm{th} i}$.

Now consider the $V_{a}=V_{0}$ case. Obviously, the solutions for $V_{a} \leq V_{0}$ (11) and $V_{a} \geq V_{0}$ (13) must give the same value for $V_{a}=V_{0}$. This leads to the condition $W_{0}\left(\alpha_{e, i}\right)=W_{-1}\left(\alpha_{e, i}\right)$, which was shown to be satisfied only for $\alpha_{e, i}=-\frac{1}{\mathrm{e}}$, and is equivalent to $U_{i}=U_{e}=1$. Then, the convective velocity for both species must be $u_{\text {ths }}$ from infinity up to the wall. This notably implies that the flux density at the wall in the field-free situation is given by $\phi_{s}=n_{0} u_{\mathrm{th} s}=n_{0} \sqrt{\frac{q T_{s}}{m_{s}}}$. This is different from the classical kinetic theory flux density $\phi_{s}=\frac{1}{4} n_{0} \bar{v}_{s}=\frac{1}{\sqrt{2 \pi}} n_{0} u_{\mathrm{th} s}$, (where $\bar{v}_{s}=\sqrt{\frac{8 q T_{s}}{\pi m_{s}}}$ is the mean thermal velocity) which is conventionally assumed for the repelled species at the wall surface in sheath models. ${ }^{1-3,8,9,13,19,26-28,32,43,44}$ We will come back to this point in the final discussion, Section VIB.

\section{B. Criterion on initial velocities}

To complete the solutions we have to determine $U_{e 0}$ and $U_{i 0}$. A criterion on these initial velocities can be found, for any probe potential $V_{a}$, by requiring a monotonic decrease of the charge density modulus $|Q|=$ $q\left|n_{i}-n_{e}\right|$ towards infinity where $V=V_{0}$; a local condition which can be expressed as $\left[\partial_{V}|Q|\right]_{V_{0}} \leq 0 .{ }^{45}$ According to our solution (10), after some algebra this inequality becomes:

$$
T_{i}\left(U_{i 0}-1\right)+T_{e}\left(U_{e 0}-1\right) \geq 0 .
$$

This relation for the initial convective velocities, which is symmetric in electron and ion terms, can be seen as a generalization of the classical Bohm criterion. ${ }^{5,11}$ By rewriting (15) in a more conventional way to express the minimal velocity for ions entering a sheath we obtain:

$$
u_{i 0} \geq \sqrt{\frac{q\left(T_{e}+T_{i}\right)-m_{e} u_{e 0}^{2}}{m_{i}}} .
$$

The Bohm criterion ${ }^{5} u_{i 0} \geq u_{\text {Bohm }}=\sqrt{\frac{q T_{e}}{m_{i}}}$ is recovered for cold ions $\left(T_{i}=0\right)$ and negligible electron inertia $\left(m_{e}=0\right)$. Persson's ${ }^{23}$ expression is obtained when ion temperature is accounted for, and the complete expression (16) of Baalrud et $a l^{46}$ is recovered when electron inertia is also included. Note that criterion (15) implies a general coupling between ion and electron initial velocities for all bias potentials.

Interestingly, the inequality (15) is directly related to the required convective kinetic energy density at infinity, $E_{\infty}$, which can be expressed for any value of the applied 
potential as follows:

$$
\begin{aligned}
E_{\infty}\left(V_{a}\right)= & n_{0}\left(\frac{1}{2} m_{i} u_{i 0}^{2}+\frac{1}{2} m_{e} u_{e 0}^{2}\right) \\
& =\frac{1}{2} q n_{0}\left(T_{i} U_{i 0}+T_{e} U_{e 0}\right) .
\end{aligned}
$$

When $V_{a}=V_{0}$, as $U_{i 0}=U_{e 0}=1,(17)$ gives

$$
E_{\infty}\left(V_{0}\right)=E_{0}=\frac{1}{2} q n_{0}\left(T_{i}+T_{e}\right) .
$$

Taking the energy difference $E_{\infty}\left(V_{a}\right)-E_{\infty}\left(V_{0}\right)$ we obtain:

$$
E_{\infty}\left(V_{a}\right)-E_{\infty}\left(V_{0}\right)=\frac{1}{2} q n_{0}\left[T_{i}\left(U_{i 0}-1\right)+T_{e}\left(U_{e 0}-1\right)\right] .
$$

Then the criterion (15) can be interpreted as follows: $E_{\infty}\left(V_{0}\right)$ corresponds to a minimum of required convective kinetic energy density at infinity. For any other value of $V_{a}$ the required kinetic energy density can only increase. Consequently, a saturation of criterion (15), i.e. taking $T_{i}\left(U_{i 0}-1\right)+T_{e}\left(U_{e 0}-1\right)=0$, corresponds to a minimization of $E_{\infty}\left(V_{a}\right)$ which then takes the constant value $E_{0}=\frac{1}{2} q n_{0}\left(T_{i}+T_{e}\right)$ for any applied potential $V_{a}$.

Furthermore, it can be verified from (27) that, by saturating (15), the electrical field intensity at the wall is minimized, and therefore that the total charge in the wall-perturbed region is also minimized. Hence the saturation of the general criterion (15) corresponds to the minimal perturbation to the plasma by the biased wall. On the basis of these arguments we postulate the criterion (15) to be always saturated. But then what determines the partition of the constant convective kinetic energy density $E_{0}$ between ions and electrons?

To clarify this question we consider the $V_{a} \leq V_{0}$ case. Saturating (15), $U_{i 0}$ can be expressed as a function of $U_{e 0}$ :

$$
U_{i 0}=1+\frac{T_{e}}{T_{i}}\left(1-U_{e 0}\right) .
$$

From (12), $U_{e 0}$ is constrained to lie in an interval $\left[0, U_{e 0}^{\max }\right]$. From (20) it is obvious that in order to minimize the ion initial velocity one should maximize $U_{e 0}$ and take $U_{e 0}=U_{e 0}^{\max }$. By doing so, the electron convective velocity at the wall is implicitly set to $u_{\text {the }}$ for any value of the applied potential $V_{a} \leq V_{0}$, and not only for $V_{a}=V_{0}$ when it must be so. One could question the choice of minimizing the initial velocity of the fieldaccelerated species at the expense of the field-repelled one. The problem can also be stated as follows: The repelled species is adsorbed at the wall by sole virtue of its random thermal motion, which does not depend on the bias potential in the framework of an isothermal model. We know from our solution that the convective velocity for both species must be $u_{\text {ths }}$ in the field-free situation. It should then remain $u_{\text {ths }}$ for any value of $V_{a}$ as long as the considered species is repelled.

The evolution of the initial ion velocity as a function of $V_{a}$, in comparison with the Bohm velocity, is shown

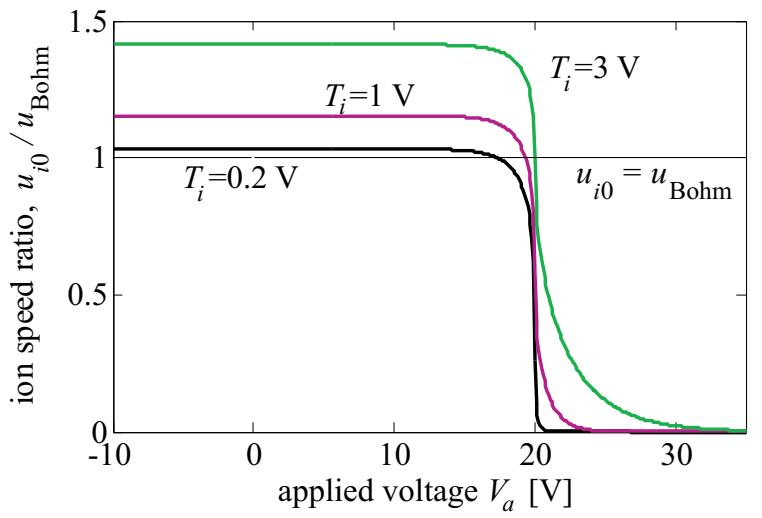

FIG. 3. Evolution of the initial ion velocity $u_{i 0}$, normalized to the Bohm velocity, for the infinite plane probe for three different values of the ion temperature $T_{i}$. Fixed parameters: Argon gas, $n_{0}=10^{17} \mathrm{~m}^{-3}, T_{e}=3 \mathrm{~V}, V_{0}=20 \mathrm{~V}$.

on Fig. 3 for three different ion temperatures. It then appears that taking the Bohm velocity as an initial velocity for ions is an acceptable approximation for low $T_{i}$ and large negative biasing.

To resume the 1D Cartesian result, we first remind that $\alpha_{e}$ and $\alpha_{i}$, from (10), are given by:

$$
\begin{gathered}
\alpha_{e}=-U_{e 0} \mathrm{e}^{-U_{e 0}-\frac{2\left(V-V_{0}\right)}{T_{e}}}, \\
\alpha_{i}=-U_{i 0} \mathrm{e}^{-U_{i 0}+\frac{2\left(V-V_{0}\right)}{T_{i}}} .
\end{gathered}
$$

The 1D Cartesian moment equations for $V_{a} \leq V_{0}$ are finally reduced to

$$
\begin{array}{r}
U_{e}=-W_{0}\left[\alpha_{e}\right] \quad \text { and } U_{i}=-W_{-1}\left[\alpha_{i}\right], \\
\text { where } U_{i 0}=1+\frac{T_{e}}{T_{i}}\left(1-U_{e 0}\right), \quad \text { and } \\
U_{e 0}=-W_{0}\left[-\mathrm{e}^{\frac{2\left(V_{a}-V_{0}\right)}{T_{e}}-1}\right] .
\end{array}
$$

The electron fluid velocity at the wall is always equal to the thermal velocity $u_{\text {the }}$. The corresponding loci of the electron and ion fluid velocities are shown on a Lambert function diagram in Fig. 4.

For $V_{a} \geq V_{0}$, the roles of electrons and ions are exchanged, accounting for charge sign:

$$
\begin{array}{r}
U_{e}=-W_{-1}\left[\alpha_{e}\right] \quad \text { and } U_{i}=-W_{0}\left[\alpha_{i}\right], \\
\text { where } U_{e 0}=1+\frac{T_{i}}{T_{e}}\left(1-U_{i 0}\right), \quad \text { and } \\
U_{i 0}=-W_{0}\left[-\mathrm{e}^{-\frac{2\left(V_{a}-V_{0}\right)}{T_{i}}-1}\right] .
\end{array}
$$

\section{Wall current-voltage characteristics}

Now that $U_{i 0}$ and $U_{e 0}$ are known, the current per unit area collected at the wall according to this 1D Cartesian 


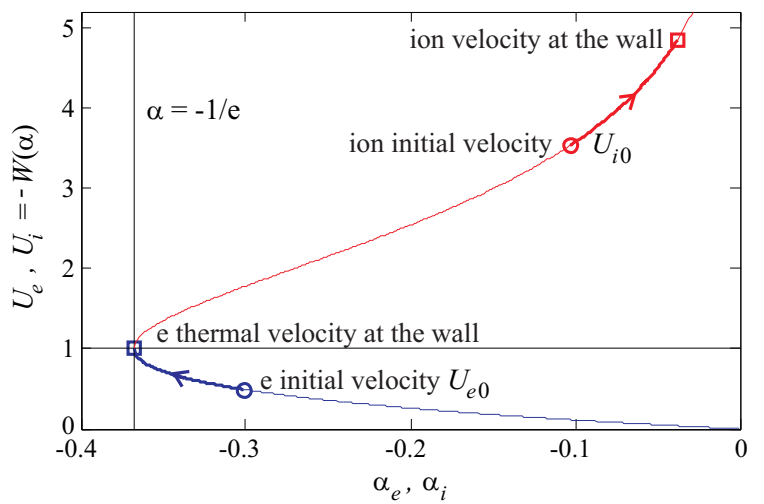

FIG. 4. Locus of the electron normalized square fluid velocity $U_{e}$ along part of the Lambert branch $W_{0}$ (blue line), and the corresponding ion locus (red line) on the Lambert branch $W_{-1}$, for the case $V_{a} \leq V_{0}$ of an infinite plane wall. The $y$ axis is inverted with respect to Fig. 2. Non-typical parameters were used here for clear presentation: $V_{0}=20 \mathrm{~V}, V_{a}=19.9 \mathrm{~V}$, $T_{e}=1 \mathrm{~V}$, and $T_{i}=0.2 \mathrm{~V}$.

solution can be calculated using

$$
J_{\mathrm{wall}}=q n_{0}\left(u_{i 0}-u_{e 0}\right) .
$$

The current-voltage $(I V)$ characteristic is a continuous solution as shown in Fig. 5, for an arbitrary set of plasma parameters. The ion and electron currents show very pronounced saturation for $V_{a} \ll V_{0}$ and $V_{a} \gg V_{0}$ respectively, as expected for an infinite plane probe. ${ }^{1,2}$ The plasma parameters were chosen as follows: The pressure of $\sim 1 \mathrm{~Pa}$ (estimated in Section I.B) is characteristic of low temperature, non-equilibrium plasmas, for which $T_{e} \sim 3 \mathrm{~V}$ is a typical value imposed by particle balance. ${ }^{28}$ The ion temperature is generally above ambient temperature $(0.025 \mathrm{~V})$, and a relatively high value, $T_{i}=0.2 \mathrm{~V}$, was chosen here to show its effect in the figures. The plasma density, $n_{0} \sim 10^{17} \mathrm{~m}^{-3}$ corresponds to a representative degree of ionisation $n_{0} / n_{\text {gas }} \sim 10^{-4}$, and the electron Debye length $\lambda_{D e}=\left(\varepsilon_{0} T_{e} /\left(n_{0} q\right)\right)^{1 / 2}$ is $0.04 \mathrm{~mm}$. The plasma potential, $V_{0}=20 \mathrm{~V}$, is an arbitrary reference level chosen here so that the floating potential $V_{f}$ is not far from $0 \mathrm{~V}$, because the sheath voltage at a floating probe is often not very different from the sheath voltage at the ground electrode. ${ }^{28}$ Figures can be re-calculated for other plasma parameters by using the analytical expressions given in the text.

The influence of $T_{i}$ on the value of the electron saturation current and on the shape of the $I V$ characteristic around $V_{a}=V_{0}$ is illustrated in Fig. 6. The curve for $T_{i}=T_{e}$ is later compared with a numerical result of Parrot et al, ${ }^{36}$ in the limit of probe radius much larger than the sheath thickness, in Fig. 15.

The expression for the floating potential $V_{f}$ of the wall is deduced by setting $J_{\text {wall }}=0$ in (23):

$$
V_{f}^{\text {wall }}=V_{0}-\frac{T_{e}}{2}(\ln A+1 / A-1),
$$

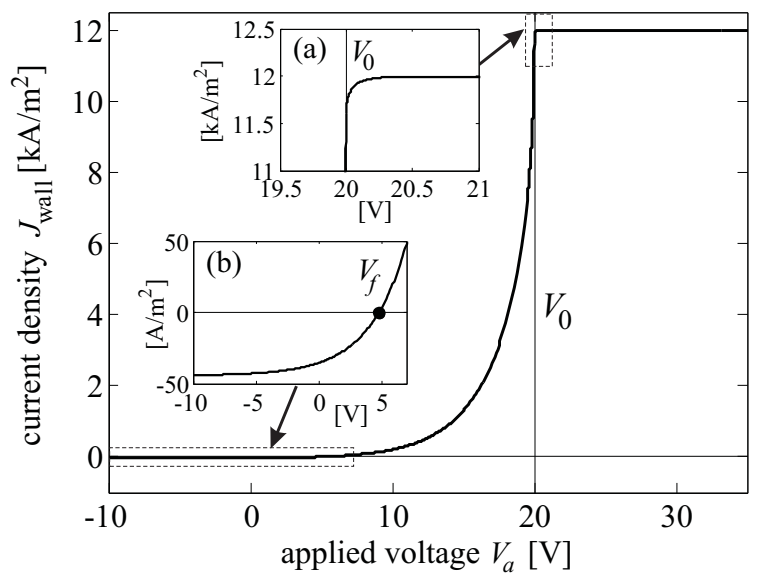

FIG. 5. Typical current-voltage characteristic for the infinite plane probe predicted by the Lambert solution, using (23). Insets show details (a) around the plasma potential $V_{0}$, where the curvature depends on $T_{i}$; and (b) around the floating potential $V_{f}$. Fixed parameters: Argon gas, $V_{0}=20 \mathrm{~V}$, $n_{0}=10^{17} \mathrm{~m}^{-3}, T_{i}=0.2 \mathrm{~V}, T_{e}=3 \mathrm{~V}$.

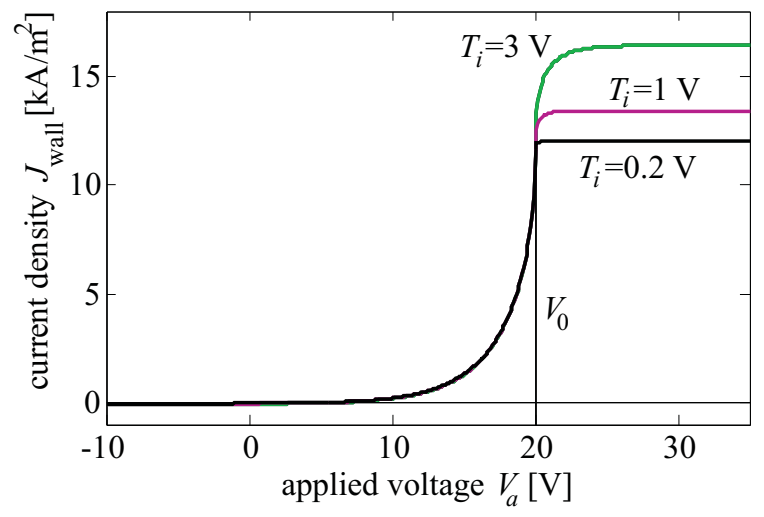

FIG. 6. Current-voltage characteristics for the infinite plane probe for three different values of the ion temperature $T_{i}$, using (23). Fixed parameters: Argon gas, $n_{0}=10^{17} \mathrm{~m}^{-3}$, $T_{e}=3 \mathrm{~V}, V_{0}=20 \mathrm{~V}$.

where $A=\frac{T_{e}\left(m_{i}+m_{e}\right)}{m_{e}\left(T_{e}+T_{i}\right)}$.

\section{Potential and electric field}

The ultimate step for a complete solution would be to obtain the spatial profiles by integrating Poisson's equation,

$$
\partial_{x}^{2} V=-\frac{q}{\varepsilon_{0}}\left(n_{i}-n_{e}\right)
$$

To our knowledge, an analytical solution cannot be found, but at least the square electric field intensity $E^{2}=\left(-\partial_{x} V\right)^{2}$ can be expressed everywhere by the following calculation: 
Multiplying (25) by $\partial_{x} V$ gives

$$
\partial_{x} V \partial_{x}^{2} V=\frac{1}{2} \partial_{x}\left[\left(\partial_{x} V\right)^{2}\right]=-\frac{q}{\varepsilon_{0}}\left(n_{i}-n_{e}\right) \partial_{x} V .
$$

Using the fluid equations for electron and ion momentum (2), and making use of particle conservation $\left(n_{e} u_{e}=\right.$ $n_{0} u_{e 0}=\phi_{e}$ and $\left.n_{i} u_{i}=n_{0} u_{i 0}=\phi_{i}\right)$, we have:

$$
\begin{array}{r}
m_{e} \phi_{e} \partial_{x} u_{e}-q n_{e} \partial_{x} V+q T_{e} \partial_{x} n_{e}=0, \\
m_{i} \phi_{i} \partial_{x} u_{i}+q n_{i} \partial_{x} V+q T_{i} \partial_{x} n_{i}=0 .
\end{array}
$$

By summing these two equations,

$$
\begin{array}{r}
-q\left(n_{i}-n_{e}\right) \partial_{x} V= \\
m_{e} \phi_{e} \partial_{x} u_{e}+q T_{e} \partial_{x} n_{e} \\
+m_{i} \phi_{i} \partial_{x} u_{i}+q T_{i} \partial_{x} n_{i} .
\end{array}
$$

Then (26) can be directly integrated to find the expression for the square electric field, the integration constant being defined by the limits at infinity:

$$
\begin{gathered}
\frac{\varepsilon_{0}}{2} E^{2}=-q T_{i}\left(n_{0}-n_{i}\right)-q T_{e}\left(n_{0}-n_{e}\right) \\
-m_{i} \phi_{i}\left(u_{i 0}-u_{i}\right)-m_{e} \phi_{e}\left(u_{e 0}-u_{e}\right) .
\end{gathered}
$$

Equations (25) or (27) can be integrated numerically starting from a point supposed far enough from the wall so that we can take as initial conditions $n_{e}=n_{i}=n_{0}$, $U_{i}=U_{i 0}, U_{e}=U_{e 0}$, and $V=V_{0}$. An initial infinitesimal gradient for $V$ is imposed and the spatial evolution of the potential is computed until the condition $V=V_{a}$ is satisfied, which corresponds to the wall. Alternatively, since we know the electric field at the wall surface from (27), we can also integrate Poisson's equation starting from the wall. Both methods give the same result, although the integration starting from the wall tends to diverge and needs much smaller integration steps. For examples, plots of computed profiles for $V_{a}$ lower, and greater, than $V_{0}$ are shown in Figs. 7(a) and (b), respectively. The ion-rich perturbed region in Fig. 7(a) is several Debye lengths wide, but much smaller than the mean free path $\lambda_{c}$, which is characteristic of a collisionless thin sheath for a classical Langmuir probe. ${ }^{47}$ The electron-rich perturbation in Fig. 7(b) is narrower and more similar to the Debye length. In Fig. 7(a), the ion initial convective velocity is slightly greater than the Bohm velocity (see Fig. 3 for $V_{a}=15 \mathrm{~V}$ ), and the electron convective velocity at the wall equals their thermal velocity ${ }^{35}$ as discussed in Section II B. Note that the electron initial convective velocity in the $V_{a}>V_{0}$ case is slightly higher than their thermal velocity $u_{\text {the }}$, as shown also by Scheiner et al. ${ }^{31}$

\section{E. Discussion of the 1D infinite plane wall case}

As already emphasized, the 1D Cartesian collisionless example above, corresponding to an infinite plane wall and a semi-infinite plasma, clearly does not apply to the physical case of a real probe immersed in a plasma. ${ }^{1}$ The wall acts more like an electrode than a probe in this case, and a pre-sheath must be considered. ${ }^{11,12,30}$ The
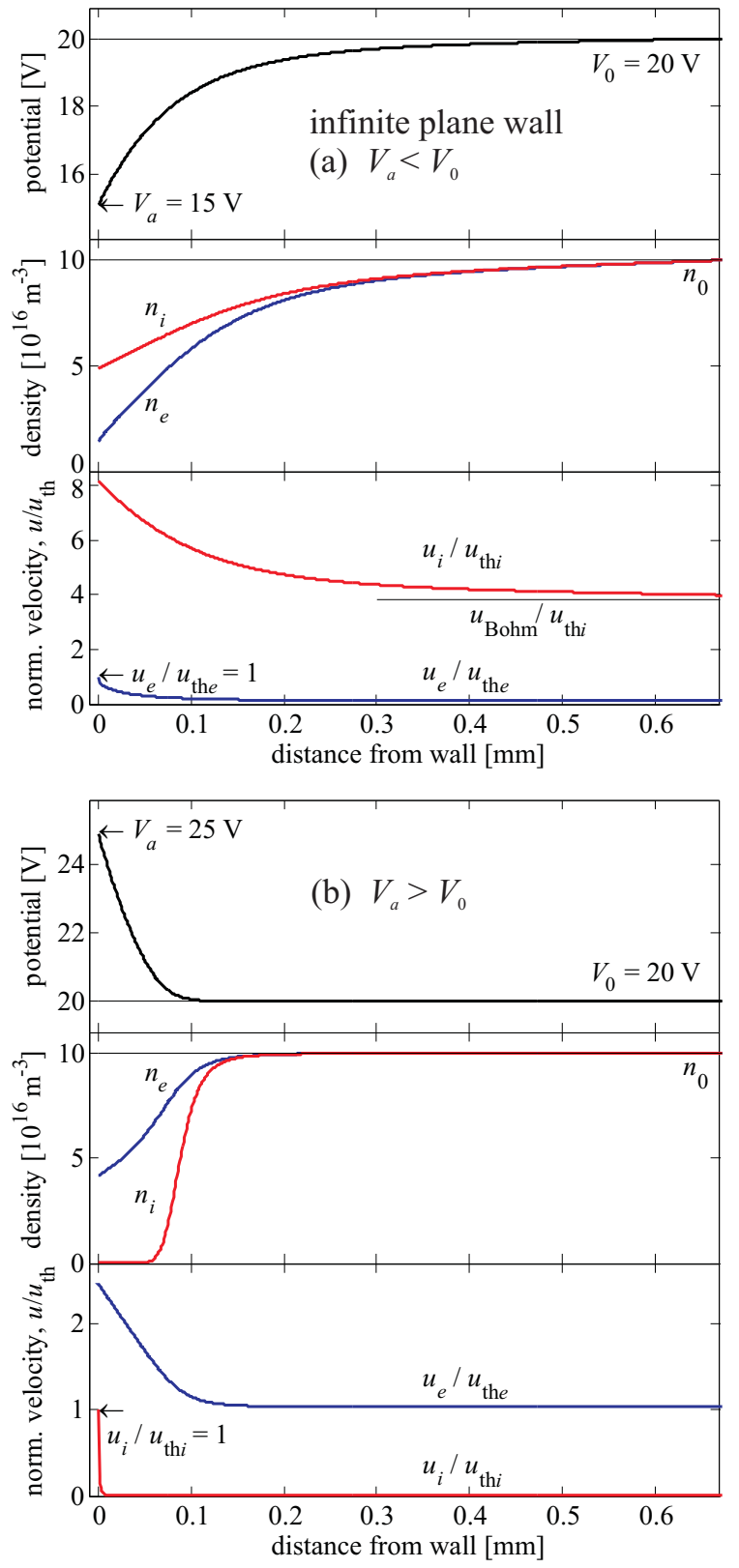

FIG. 7. Potential profile adjacent to an infinite plane wall, ion and electron densities, and velocities normalized by their thermal velocity $\left(692 \mathrm{~m} / \mathrm{s}\right.$ and $7.26 \cdot 10^{5} \mathrm{~m} / \mathrm{s}$ respectively), (a) for $V_{a}=15 \mathrm{~V}<V_{0}=20 \mathrm{~V}$; and (b) for $V_{a}=25 \mathrm{~V}>V_{0}=$ 20 V. Same plasma parameters as for Fig. 5.

fundamental drawback of this 1D Cartesian collisionless solution is that conservation of particle flux requires a non-zero convective velocity plasma source from infinity (Fig. 1(a)), whereas for real finite-sized bodies, particle flux conservation imposes a limit of zero convective velocity from infinity (Fig. 1(b)), as will be shown in the next section. In many conventional 1D models, conditions for finite-sized bodies are nevertheless imposed at infinity $\left(n_{s}=n_{0}, V=V_{0}, U_{s}=0\right)$, and as such, the 1D Cartesian particle conservation is violated, unless ionization is 
included in a collisional pre-sheath. ${ }^{6}$ As a consequence there is an artificial separation of space into a collisionless sheath, with a 1D Cartesian constant particle flux, and a collisional pre-sheath where the flux is increasing up to the sheath entrance (Fig. 1(c)), with considerable difficulties to match the two solutions. This problem is considered further in section $\mathrm{V}$ in the framework of the big radius probe approximation.

\section{1D RADIAL GEOMETRY FOR CYLINDRICAL PROBES}

For radial symmetry, the fluid equations for electron and ion momentum are found to be identical to the 1D Cartesian situation (3):

$$
\begin{gathered}
\frac{1}{2} \partial_{r} U_{e}-\frac{1}{T_{e}} \partial_{r} V+\partial_{r} \ln \left(n_{e}\right)=0, \\
\frac{1}{2} \partial_{r} U_{i}+\frac{1}{T_{i}} \partial_{r} V+\partial_{r} \ln \left(n_{i}\right)=0 .
\end{gathered}
$$

In cylindrical geometry, however, the steady state radial particle flux conservation is expressed as $\frac{1}{r} \partial_{r}\left(r n_{s} u_{s}\right)=0$, hence

$$
2 \pi r n_{e} u_{e}=\Gamma_{e}, \quad \text { and } \quad 2 \pi r n_{i} u_{i}=\Gamma_{i},
$$

where $\Gamma_{e, i}$ is the constant particle flux per unit length of the probe, for electrons and ions respectively. As for the 1D Cartesian case, we set at infinity an equal density $n_{0}$ for electrons and ions, and the plasma potential $V_{0}$. This being stated, it is clear from (29) that both electron and ion fluid velocities must tend toward zero as $r$ tends toward infinity to ensure a finite flux (see Fig. 1(b)). Then, the integration of (28), making use of the boundary conditions at infinity, leads to

$$
\begin{aligned}
& n_{e}=n_{0} \exp \left(\frac{-U_{e}}{2}+\frac{V-V_{0}}{T_{e}}\right), \\
& n_{i}=n_{0} \exp \left(\frac{-U_{i}}{2}-\frac{V-V_{0}}{T_{i}}\right) .
\end{aligned}
$$

Because the fluxes $\Gamma_{e, i}$ can no longer be defined by boundary conditions at infinity, we use instead a definition at the cylindrical probe surface located at $r=R_{p}$ :

$$
2 \pi R_{p} n_{e a} u_{e a}=\Gamma_{e}, \quad \text { and } \quad 2 \pi R_{p} n_{i a} u_{i a}=\Gamma_{i},
$$

$u_{e a}$ and $u_{i a}$ being the electron and ion fluid velocities at the probe and $n_{e a}$ and $n_{i a}$ the corresponding densities according to (30), with the potential of the probe being defined as $V_{a}$. We further redefine the normalized square velocities at the probe as

$$
U_{e a}=\left(\frac{u_{e a}}{u_{\mathrm{the}}}\right)^{2} \text { and } \quad U_{i a}=\left(\frac{u_{i a}}{u_{\mathrm{th} i}}\right)^{2} .
$$

Then the combination of (29) to (32) leads to the solutions for the square velocities $U_{e}$ and $U_{i}$, which take the following forms:

$$
\begin{gathered}
U_{e}=-W\left[-\frac{R_{p}^{2} U_{e a}}{r^{2}} \mathrm{e}^{-U_{e a}+\frac{2\left(V_{a}-V\right)}{T_{e}}}\right]=-W\left[\alpha_{e}\right], \\
U_{i}=-W\left[-\frac{R_{p}^{2} U_{i a}}{r^{2}} \mathrm{e}^{-U_{i a}-\frac{2\left(V_{a}-V\right)}{T_{i}}}\right]=-W\left[\alpha_{i}\right]
\end{gathered}
$$

As in the $1 \mathrm{D}$ Cartesian case, both $U_{e}$ and $U_{i}$ are real and positive, so they can only be described by the $W_{0}$ and $W_{-1}$ branches of the Lambert function, and $\alpha_{e, i}$ must lie in the interval $\left[-\frac{1}{\mathrm{e}}, 0\right]$. We still assume for this radial case a monotonic variation of the electric potential with $r$. In the 1D Cartesian geometry, the monotonicity of $V(x)$ implies the monotonicity of the Lambert function arguments $\alpha_{e, i}(x)$, so that the analysis of the conditions $\alpha_{e, i}(x) \in\left[-\frac{1}{\mathrm{e}}, 0\right]$ concerns only the boundary conditions at infinity and at the wall surface, as shown in Appendix A. In cylindrical geometry this is no longer true, because of the direct dependence on $r$ of $\alpha_{e, i}(r)$. In fact, taking the spatial derivative of $\alpha_{e}$ and $\alpha_{i}$ one finds:

$$
\begin{aligned}
\partial_{r} \alpha_{e} & =\frac{-2 \alpha_{e}}{T_{e}}\left(\frac{T_{e}}{r}+\partial_{r} V\right), \\
\partial_{r} \alpha_{i} & =\frac{-2 \alpha_{i}}{T_{i}}\left(\frac{T_{i}}{r}-\partial_{r} V\right) .
\end{aligned}
$$

To understand the meaning of this, we focus in the following on the $V_{a}<V_{0}$ case. Upon the monotonicity hypothesis, the potential continuously increases with $r$ so that $\partial_{r} V>0$. For electrons, (34) never vanishes for $\partial_{r} V>0$ and the argument $\alpha_{e}(r)$ is still monotonic. As in the 1D Cartesian model, the condition $\alpha_{e} \in\left[-\frac{1}{\mathrm{e}}, 0\right]$ implies that the electron convective velocity at the probe lies in the interval $\left[0, u_{\text {the }}\right]$. We have shown in the $1 \mathrm{D}$ Cartesian Section II.B that taking $u_{e a}=u_{\text {the }}$ corresponds to a minimal perturbation to the plasma by the biased wall. We postulate in the following that this remains true in radial geometry, or, in other words, that the adsorption mechanism of the repelled species at the probe does not depend on the geometry considered. Then, setting $u_{e a}=u_{\text {the }}$ the electron density at the probe surface is known using (30):

$$
n_{e a}=n_{0} \mathrm{e}^{-\frac{1}{2}} \mathrm{e}^{\frac{V_{a}-V_{0}}{T_{e}}} .
$$

For ions, according to (34) the argument $\alpha_{i}$ reaches its minimum at a radius $R_{S}$ for which $\left[\partial_{r} V\right]_{R_{S}}=T_{i} / R_{S}$. Consider then the ion velocity evolution (33) starting from infinity toward the probe, as shown schematically in Fig. 8. For large $r, U_{i}$ tends toward zero and can then only be represented by the first branch of Lambert functions, $W_{0}$. Approaching the probe, $\alpha_{i}$ decreases, and the ion velocity increases up to the minimum of $\alpha_{i}$ when $r=R_{S}$. Beyond this point, as $\alpha_{i}$ now increases, the ion velocity would decrease if it were to remain represented by $W_{0}$. As, on the contrary, we expect the ion velocity to continuously increase even for $r<R_{S}$, this implies that the solution must switch to the second Lambert branch, 


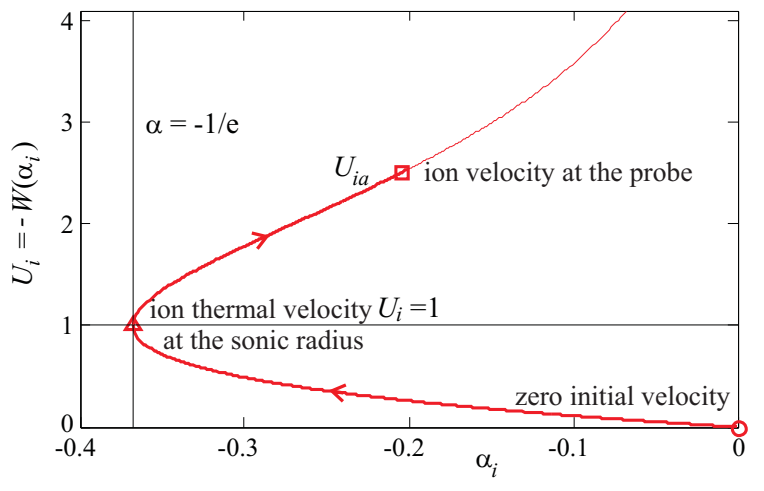

FIG. 8. Locus of the ion normalized square fluid velocity for the case $V_{a} \leq V_{0}$ of a cylindrical probe, showing the zero initial velocity (circle), the ion thermal velocity at the sonic radius (triangle), and the ion velocity at the probe (square). The $y$ axis is inverted with respect to Fig. 2. The locus of the electron velocity (not shown) also begins at the origin and traverses the branch $W_{0}$, but ends on the probe at the electron thermal velocity, point $\left[-\frac{1}{\mathrm{e}}, 1\right]$.

$W_{-1}$. However, to ensure a continuous solution, the continuity between the two branches $W_{0}$ and $W_{-1}$ must be satisfied at $r=R_{S}$. This can only be achieved by setting $\alpha_{i}\left(R_{S}\right)=-\frac{1}{\mathrm{e}}$, which implies that the ion convective velocity at $R_{S}$ must be $u_{\mathrm{th} i}$. $R_{S}$ can then be defined as the ion sonic radius (this is not the same as the ion acoustic speed associated with Bohm's velocity). Clearly, for probe theories with cold ions $\left(T_{i}=0\right)$, the ion sonic radius could have no meaning.

An interesting question arises when the applied potential $V_{a}$ approaches $V_{0}$. Clearly, for the field-free case $V_{a}=V_{0}$, a sonic radius $R_{S}$ can no longer be defined because $\partial_{r} V=0$ everywhere. Then, there must be a limit value $V_{a}=V_{\lim }$ for which $R_{S}$ coincides with $R_{p}$, implying that the ion convective velocity at the probe is $u_{\text {thi }}$ for this case. Above $V_{\lim }$ the ion convective velocity could be thought to fall below $u_{\text {thi } i}$. On the other hand, according to the 1D Cartesian solution, for the field-free situation $\left(V_{a}=V_{0}\right)$ we expect the ion and electron convective velocities at the probe to be $u_{\text {th } i \text {,the }}$, and we have indeed already postulated that this remains true in the radial case by setting $u_{e a}=u_{\text {the }}$ above. This then implies that the convective velocities of ions and electrons in the zone between the two limit potentials $V_{\lim }$ (one for $V_{a}<V_{0}$ when $\left[\partial_{r} V\right]_{R_{p}}=T_{i} / R_{p}$, and one for $V_{a}>V_{0}$ when $\left.\left[\partial_{r} V\right]_{R_{p}}=-T_{e} / R_{p}\right)$ are to be constant at $u_{\mathrm{th} i}$ and $u_{\text {the }}$, respectively.

Under this assumption, in this "constant velocity zone", both ion and electron densities at the probe are known, using (30), and can be expressed for any probe radius $R_{p}$ as:

$$
\begin{gathered}
n_{e a}=n_{0} \mathrm{e}^{-\frac{1}{2}} \mathrm{e}^{\frac{V_{a}-V_{0}}{T_{e}}}, \\
n_{i a}=n_{0} \mathrm{e}^{-\frac{1}{2}} \mathrm{e}^{-\frac{V_{a}-V_{0}}{T_{i}}} .
\end{gathered}
$$

Going back to the more general case for which an ion
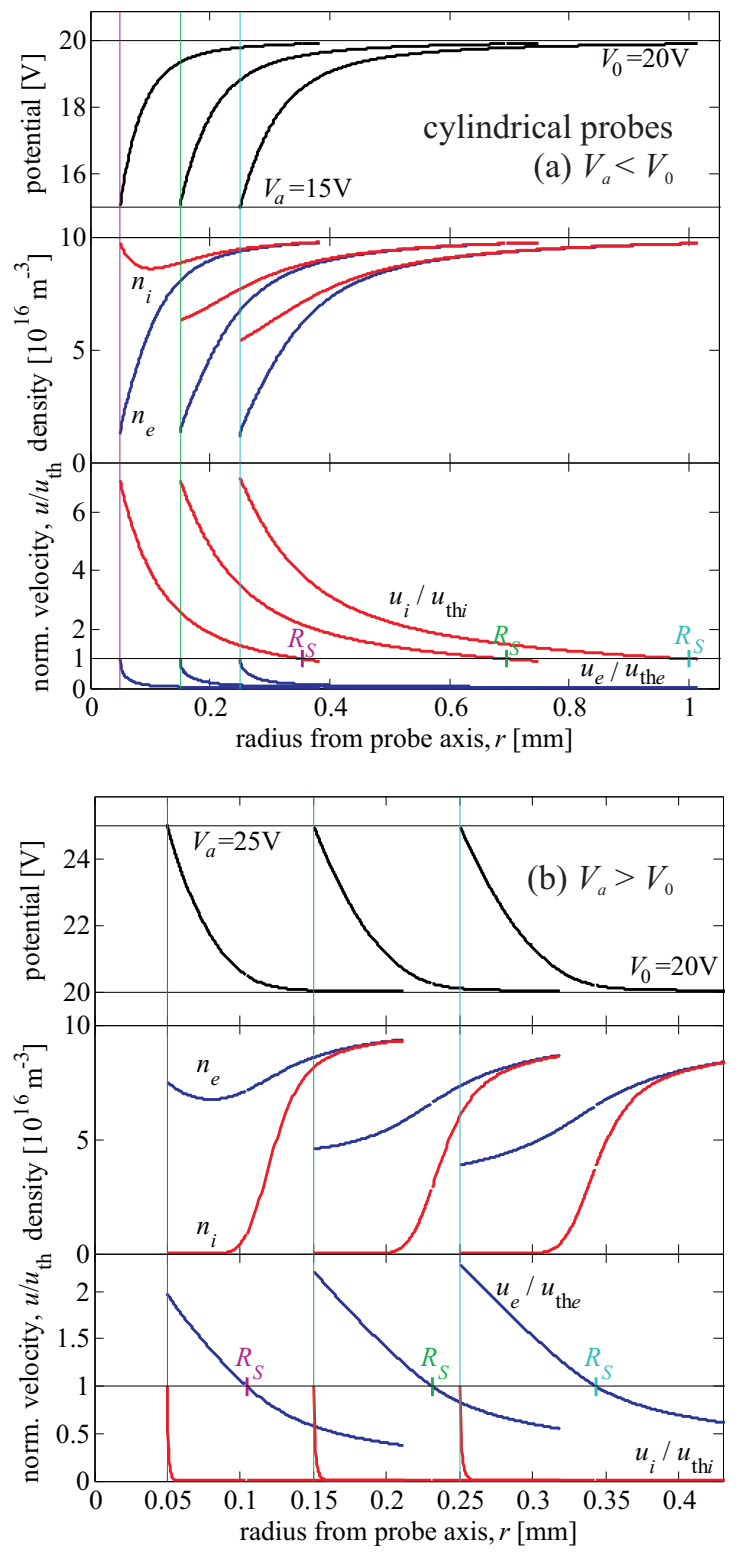

FIG. 9. Cylindrical probe 1D radial profiles of potential, density, and convective velocity normalized to the thermal velocity, (a) for probe voltage $V_{a}=15 \mathrm{~V}<V_{0}=20 \mathrm{~V}$, and (b) for probe voltage $V_{a}=25 \mathrm{~V}>V_{0}=20 \mathrm{~V}$, for three cylindrical probe radii $R_{p}=0.05,0.15$ and $0.25 \mathrm{~mm}$ (indicated by the vertical lines). The ion sonic radius $R_{S}$ in (a), and the electron sonic radius $R_{S}$ in (b), is also shown for each probe. Fixed parameters: Argon gas, $n_{0}=10^{17} \mathrm{~m}^{-3}, T_{i}=0.2 \mathrm{~V}$, $T_{e}=3 \mathrm{~V}$.

sonic radius can be defined $\left(V_{a}<V_{\lim }<V_{0}\right)$, the electron convective velocity at the probe $\left(r=R_{p}\right)$ being known to be $u_{\text {the }}$, and the ion convective velocity at the sonic radius $\left(r=R_{S}\right)$ to be $u_{\text {th } i}$, we can rewrite (33) to obtain:

$$
U_{e}=-W_{0}\left[-\frac{R_{p}^{2}}{r^{2}} \mathrm{e}^{\frac{2\left(V_{a}-V\right)}{T_{e}}-1}\right],
$$




$$
\begin{array}{cc}
U_{i}=-W_{0}\left[-\frac{R_{S}^{2}}{r^{2}} \mathrm{e}^{\frac{-2\left(V_{S}-V\right)}{T_{i}}-1}\right], \quad V \geq V_{S}, \\
U_{i}=-W_{-1}\left[-\frac{R_{S}^{2}}{r^{2}} \mathrm{e}^{\frac{-2\left(V_{S}-V\right)}{T_{i}}-1}\right], \quad V \leq V_{S} .
\end{array}
$$

$V_{S}$ is the value of the electric potential at the sonic radius $R_{S}$, and both parameters are to be determined. Recalling that these are defined by the following conditions on the electric potential:

$$
\left[\partial_{r} V\right]_{R_{S}}=\frac{T_{i}}{R_{S}}, \quad \text { and } \quad V\left(R_{S}\right)=V_{S},
$$

and that in 1D radial coordinates Poisson's equation takes the form:

$$
\frac{1}{r} \partial_{r}\left(r \partial_{r} V\right)=\frac{1}{r} \partial_{r} V+\partial_{r}^{2} V=-\frac{q}{\varepsilon_{0}}\left(n_{i}-n_{e}\right),
$$

$R_{S}$ and $V_{S}$ can be determined by requiring that the solution of Poisson's equation satisfies the boundary conditions:

$$
V\left(R_{p}\right)=V_{a}, \quad \text { and } \quad V(r \rightarrow \infty)=V_{0} .
$$

As far as we know, no analytical solution for Poisson's equation can be found, as also in the 1D Cartesian case, and we have to perform numerical integrations starting from $R_{S}$. If the correct pair $\left(R_{S}, V_{S}\right)$ is used as initial conditions, the integration of (39) towards the probe surface (decreasing $r$ ) will respect the boundary condition $V\left(R_{p}\right)=V_{a}$, while the integration towards infinity (increasing $r$ ) must lead to the limiting value $V_{0}$. The potential profile can then be computed iteratively (see Appendix B 2) in order to simultaneously satisfy these two boundary conditions. Note that, apart from a numerical determination, the value for $V_{S}$ can be analytically restricted to quite narrow intervals on the basis of simple arguments concerning the charge density and the density gradients at $r=R_{S}$, as shown in Appendix B 1 .

As a first illustrative result we show on figures 9(a) and (b) the ion and electron density profiles computed for a same value of applied potential $V_{a}\left(V_{a}<V_{0}\right.$ for figure $9(\mathrm{a})$ and $V_{a}>V_{0}$ for (b)), for three probe radii. We first remark that, although the probe radii vary from $0.05 \mathrm{~mm}$ to $0.25 \mathrm{~mm}$, the widths of the ion-rich perturbation in Fig. 9(a) are all several Debye lengths thick. This is much narrower than the mean free path $\lambda_{c}$, and is characteristic of a collisionless thin sheath for a classical Langmuir probe, ${ }^{47}$ qualitatively similar to the $1 D$ Cartesian case in Fig. 7(a). The electron-rich perturbation in Fig. 9(b) is again narrower and more similar to the Debye length. It is generally found that the sonic distance $\left(R_{S}-R_{p}\right)$ increases with increasing probe radius.

A second observation concerns the non-monotonic ion density profiles on figure 9(a) which appear for small probe radius and which are expected according to previous works, notably Laframboise. ${ }^{32}$ Note that the profiles of voltage and convective velocity remain monotonic, even when the charge densities are not. It is shown here that the same effect is expected for electrons (figure 9(b))

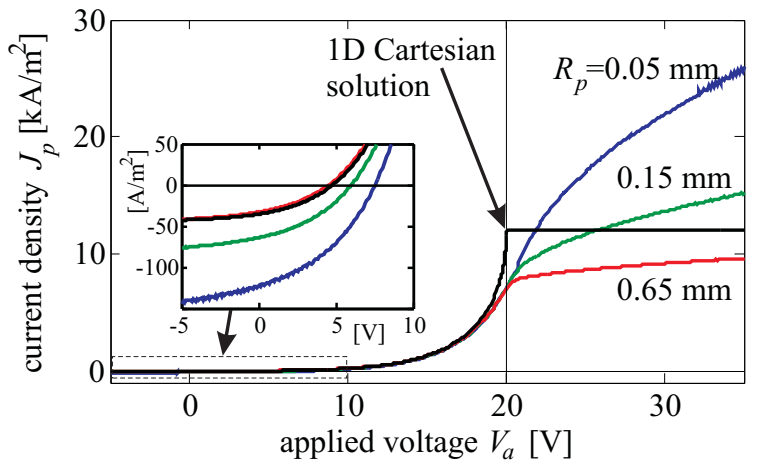

FIG. 10. Current density-voltage characteristics for three cylindrical probe radii, $R_{p}=0.05,0.15$ and $0.65 \mathrm{~mm}$, and for the 1D Cartesian solution from Fig. 5. The inset shows the ion non-saturation and floating potentials. Fixed parameters: Argon gas, $n_{0}=10^{17} \mathrm{~m}^{-3}, T_{i}=0.2 \mathrm{~V}, T_{e}=3 \mathrm{~V}$, $V_{0}=20 \mathrm{~V}$.

when $V_{a}>V_{0}$. It should also be noted that the charge separation at the ion sonic radius $R_{S}$ is very small (typically $0.01-0.1 \%$ ) for $V_{a}<V_{0}$, and this remains true up to values of $V_{a}$ very close to $V_{0}$. On the other hand, when $V_{a}>V_{0}$ the charge separation at the electron sonic radius $R_{S}$ is large (typically $50 \%$ ) even for large positive bias. It then appears that if the ion sonic radius $R_{S}$ could be thought of as a possible definition of a quasi-neutral sheath edge in the $V_{a}<V_{0}$ case (at least for $V_{a} \ll V_{0}$ ), it is not generalizable to the $V_{a}>V_{0}$ case.

\section{A. IV characteristics for cylindrical probes}

Figure 10 shows the predicted $I V$ characteristics for three different probe diameters. The results are presented as collected current per unit area $\left(\mathrm{A} / \mathrm{m}^{2}\right)$ for comparison. The noise in the characteristics, especially for the smallest probe, is due to the numerical resolution of $R_{S}$. The first important result concerns the observation of non-saturating electron and ion currents. The two-fluid solution presented here accounts for this experimental fact, which arises due to an increase in $R_{S}$ (Appendix B 3), without reference to orbital-motionlimited (OML) theory. ${ }^{2,19,21,27,48-50}$ Furthermore, the non-saturation is more pronounced for small probe radii, also as observed. ${ }^{32}$ The floating potential depends on the probe radius, as can be seen in the inset. It can generally be expressed as

$$
V_{f}^{\mathrm{cyl}}=V_{0}-\frac{T_{e}}{2} \ln \left[\frac{m_{i}}{m_{e}} \frac{T_{e}}{T_{i}} \frac{R_{p}^{2}}{R_{S}^{2}} \mathrm{e}^{\frac{2\left(V_{S}-V_{0}\right)}{T_{i}}}\right] .
$$

Fig. 10 also shows the $I V$ characteristic predicted by the 1D Cartesian solution from Fig. 5. Clearly, the large cylindrical probe characteristic (for example, $R_{p}=0.65 \mathrm{~mm}$ in Fig. 10) does not seem to tend toward the $1 \mathrm{D}$ Cartesian result as could be expected. This 


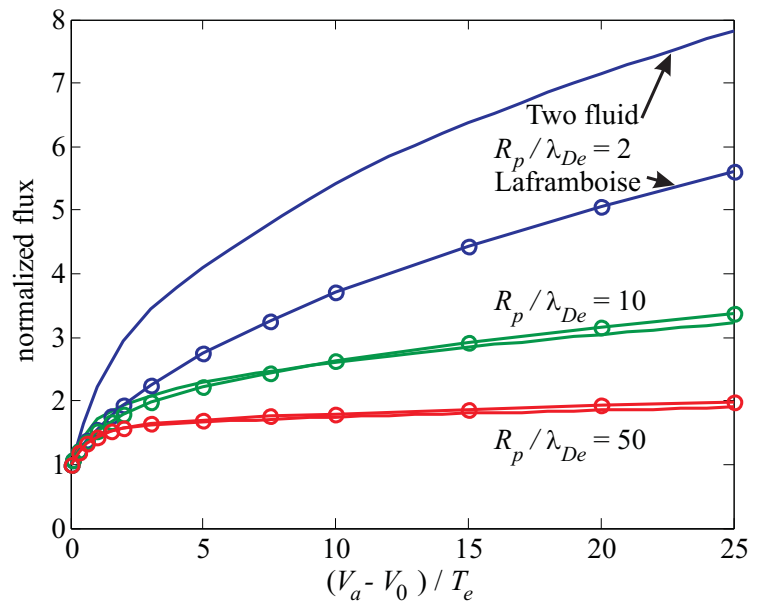

FIG. 11. Comparison between the present model and graphical results of Laframboise ${ }^{32}$ (Fig. 39), for the flux of attracted species (normalized to the value at the plasma potential $V_{0}$ ) $v s$ the dimensionless probe potential $\left(V_{a}-V_{0}\right) / T_{e}$. Lines and circles for the Laframboise results; plain lines for the present model. Results are shown for three ratios of probe radius to electron Debye length, $R_{p} / \lambda_{D e}=2,10,50$, calculated for $T_{e}=T_{i}=3 \mathrm{~V}$.

contradiction will be explained in Section V in the framework of the big radius probe approximation.

Figure 11 shows the flux of attracted species calculated for the special case $T_{i}=T_{e}$, in order to compare with graphical results of Laframboise. ${ }^{32}$ The agreement is good for the larger probes (probe radius to electron Debye length, $\left.R_{p} / \lambda_{D e}=10,50\right)$, although the nonsaturation for the small probe $\left(R_{p} / \lambda_{D e}=2\right)$ is more pronounced for the present model.

In order to fully exploit this radial solution for the analysis of measured characteristics, one has to know the dependence of the sonic radius $R_{S}$ and of the sonic potential $V_{S}$ with the applied voltage $V_{a}$. However, we show in Appendix B 4 how the main plasma parameters, $n_{0}$ and $T_{e}$, can be conveniently extracted from experimentally-measured characteristics, upon the assumption of a constant ion current contribution between floating and plasma potentials.

\section{1D RADIAL GEOMETRY FOR SPHERICAL PROBES}

The analysis of the spherical probe can be carried out by following the same method as for the cylindrical probe. The $1 \mathrm{D}$ radial expressions are similar and one has just to perform a $r \rightarrow r^{2}$ substitution to switch from the cylindrical case to the spherical one. Attention has nevertheless to be paid to the fact that the sonic radius $R_{S}$ is now defined by the relation:

$$
\left[\partial_{r} V\right]_{R_{S}}= \pm \frac{2 T_{i, e}}{R_{S}} .
$$

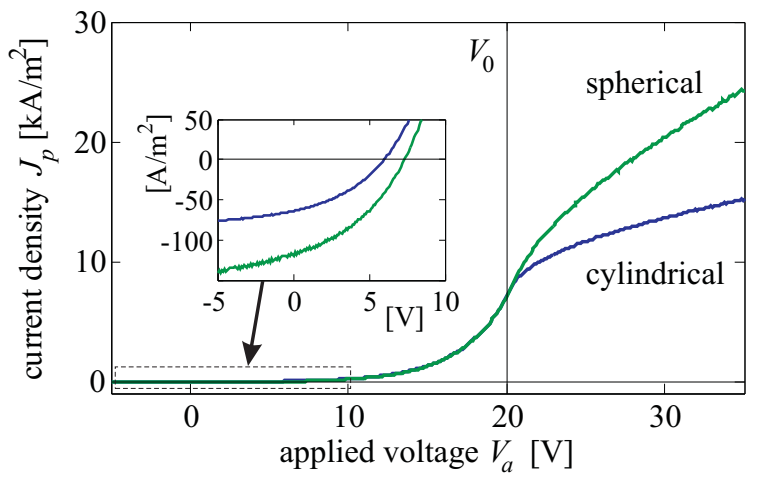

FIG. 12. Current density-voltage characteristics predicted for a cylindrical and a spherical probe, both with radius $R_{p}=$ $0.15 \mathrm{~mm}$. The inset shows the ion non-saturation and floating potentials. Fixed parameters: Argon gas, $n_{0}=10^{17} \mathrm{~m}^{-3}$, $T_{i}=0.2 \mathrm{~V}, T_{e}=3 \mathrm{~V}, V_{0}=20 \mathrm{~V}$.

Figure 12 shows the $I V$ characteristics (current per unit area) predicted for a cylindrical probe $\left(R_{p}=\right.$ $0.15 \mathrm{~mm}$ ) and a spherical probe of same radius. It can be seen that the non-saturation of spherical probes is more pronounced than for cylindrical ones, as seen in experimental observation and numerical results. ${ }^{32}$

\section{1D RADIAL GEOMETRY IN THE LIMIT OF BIG RADIUS PROBES}

Intuitively, the cylindrical 1D radial solution should tend toward the 1D Cartesian solution when the probe radius $R_{p}$ becomes very large compared to the perturbed region. It can be seen on figure 10 that when the probe diameter is increased the $I V$ characteristic becomes more and more saturated for large biasing, as expected. However, the values of the ion and electron saturation currents are higher for the 1D Cartesian solution than for the cylindrical one with large $R_{p}$. This apparent contradiction arises from an incompatibility between the boundary conditions taken for the velocities at infinity in the two geometries, which can be explained as follows:

For both models, an equal density $n_{0}$ for ions and electrons and a uniform electric potential $V_{0}$ can certainly be imposed at infinity. The difference then comes from the particle conservation equations

$$
\begin{aligned}
n_{s} u_{s}=\phi_{s} & \text { for 1D Cartesian, } \\
2 \pi r n_{s} u_{s}=\Gamma_{s} & \text { for 1D cylindrical. }
\end{aligned}
$$

At infinity, where $n_{s}=n_{0}, u_{s}$ cannot vanish for the $1 \mathrm{D}$ Cartesian case because the constant particle flux densities $\phi_{s}$ must be non-zero (Fig. 1(a)). On the other hand, to ensure constant, finite values of $\Gamma_{s}$, the velocity must tend to zero at infinity in the cylindrical situation (Fig. 1(b)). Therefore, in the limit of large $R_{p}$, the $1 \mathrm{D}$ Cartesian result can never be recovered because, however large the probe radius, the initial velocities at infinity must be zero. Another consequence of vanishing initial 
velocities is that the particle densities at the probe for $V_{a}=V_{0}$ are $n_{0} \mathrm{e}^{-\frac{1}{2}}$ in the cylindrical analysis (see (35)) and not $n_{0}$ as in the 1D Cartesian model.

We therefore focus now on the big radius probe problem. The fluid equations for electron and ion momentum (28) do not depend on $R_{p}$ and will not be changed in the framework of the large probe approximation. Then equations (30) remain valid:

$$
\begin{aligned}
n_{e} & =n_{0} \exp \left(\frac{-U_{e}}{2}+\frac{V-V_{0}}{T_{e}}\right), \\
n_{i} & =n_{0} \exp \left(\frac{-U_{i}}{2}-\frac{V-V_{0}}{T_{i}}\right) .
\end{aligned}
$$

Consider now particle conservation in cylindrical $1 \mathrm{D}$ radial systems for a distance $x$ above the probe surface:

$$
r n_{s} u_{s}=\left(R_{p}+x\right) n_{s} u_{s}=\frac{\Gamma_{s}}{2 \pi}=R_{p} n_{s a} u_{s a},
$$

where $n_{s a}$ and $u_{s a}$ are respectively the density and the velocity of the considered species at the probe surface. If we take the probe radius to be very large with respect to the sheath length scale, then $x$ can be neglected with regard to $R_{p}$ in (42) and we directly obtain the 1D Cartesian particle conservation law in this limit:

$$
n_{s} u_{s}=n_{s a} u_{s a}=\phi_{s}
$$

Boundary conditions are necessary to solve the problem as in the 1D Cartesian section, but $x$ cannot extend to infinity in the framework of this approximation. Instead, we have to state an upper boundary for our solution at a given position $x_{\mathrm{sh}} \ll R_{p}$. The only condition we will require at this radius is an equal density $n_{\mathrm{sh}}$ for ions and electrons, by analogy with the sheath defining the boundary of quasi-neutrality with respect to the pre-sheath in conventional models. ${ }^{5,11,12}$ By doing so, we employ the classical notions of sheath and pre-sheath, but it has to be noted that conditions other than quasi-neutrality at the sheath edge could, or even should, be envisaged.

\section{A. The sheath in the limit of big radius probes}

The potential at $x_{\mathrm{sh}}$ is set to be $V_{\mathrm{sh}}$ while the initial electron and ion velocities at the sheath edge are $u_{e s h}$ and $u_{i s h}$ (Fig. 1(c)). With these definitions, the combination of (30) and (43) gives the solutions

$$
\begin{gathered}
U_{e}=-W\left[-\frac{n_{\mathrm{sh}}^{2}}{n_{0}^{2}} U_{e s h} \mathrm{e}^{-\frac{2\left(V-V_{0}\right)}{T_{e}}}\right], \\
U_{i}=-W\left[-\frac{n_{\mathrm{sh}}^{2}}{n_{0}^{2}} U_{i \mathrm{sh}} \mathrm{e}^{\frac{2\left(V-V_{0}\right)}{T_{i}}}\right],
\end{gathered}
$$

where we have reintroduced the normalized initial square velocities $U_{\text {esh }}=\left(u_{\text {esh }} / u_{\text {the }}\right)^{2}$ and $U_{i \mathrm{sh}}=\left(u_{i \mathrm{sh}} / u_{\mathrm{th} i}\right)^{2}$. In order to be self-consistent, (44) must lead to $U_{e}=U_{\text {esh }}$

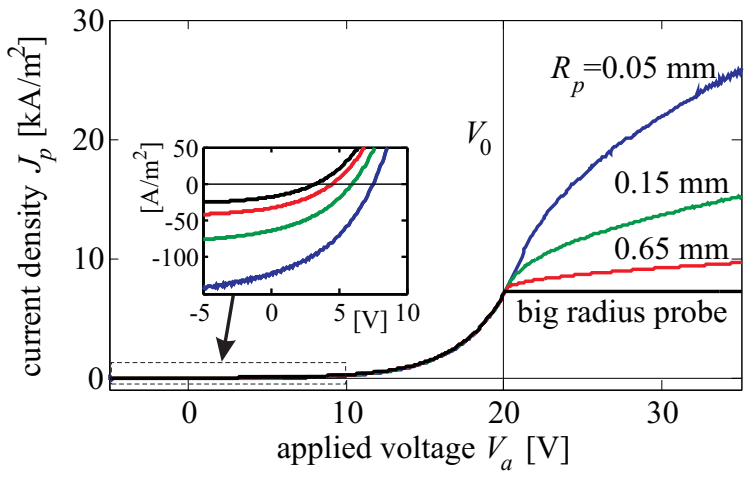

FIG. 13. Current density-voltage characteristics predicted for three different cylindrical probe radii $(0.05,0.15$ and $0.65 \mathrm{~mm})$ and for the big radius probe approximation. The inset shows the ion non-saturation. Fixed parameters: Argon gas, $n_{0}=$ $10^{17} \mathrm{~m}^{-3}, T_{i}=0.2 \mathrm{~V}, T_{e}=3 \mathrm{~V}, V_{0}=20 \mathrm{~V}$.

and $U_{i}=U_{i \mathrm{sh}}$ for $V=V_{\mathrm{sh}}$. From these two conditions we obtain

$$
\begin{array}{r}
V_{\mathrm{sh}}=V_{0}-\frac{T_{e} T_{i}\left(U_{i \mathrm{sh}}-U_{e \mathrm{esh}}\right)}{2\left(T_{e}+T_{i}\right)}, \\
n_{\mathrm{sh}}=n_{0} \mathrm{e}^{-\frac{T_{i} U_{i \mathrm{sh}}+T_{e} U_{e s h}}{2\left(T_{e}+T_{i}\right)}} .
\end{array}
$$

As in the 1D Cartesian model, we express the condition for a continuous variation of the net charge density at the sheath edge as:

$$
\left[\partial_{V} Q\right]_{V_{\mathrm{sh}}}=\left[\partial_{V}\left(n_{i}-n_{e}\right)\right]_{V_{\mathrm{sh}}} \leq 0,
$$

which according to (30) and (44-46), after some algebra, gives again the general criterion (15):

$$
T_{i}\left(U_{i \mathrm{sh}}-1\right)+T_{e}\left(U_{e s h}-1\right) \geq 0 .
$$

Now consider the case for which $V_{a}<V_{0}$, the ion-rich sheath. As explained in the 1D Cartesian section, we then take the initial normalized square velocity for electrons $U_{e s h}$ to be $U_{\text {esh }}^{\max }$, its maximum value. Therefore, taking (15) to be saturated gives

$$
U_{i \mathrm{sh}}=1+\frac{T_{e}}{T_{i}}\left(1-U_{e \mathrm{sh}}^{\max }\right) .
$$

Then, according to this expression for $U_{i s h},(45)$ and (46) become

$$
\begin{gathered}
V_{\mathrm{sh}}=V_{0}-\frac{T_{e}}{2}\left(1-U_{e \mathrm{sh}}^{\max }\right), \\
n_{\mathrm{sh}}=n_{0} \mathrm{e}^{-\frac{1}{2}}
\end{gathered}
$$

with

$$
U_{e s h}^{\max }=\mathrm{e}^{\frac{2\left(V_{a}-V_{0}\right)}{T_{e}}} .
$$

Interestingly, in the framework of this big radius probe approximation, we find the classical result of a potential drop (with regard to plasma potential) at the sheath edge 
of approximatively $T_{e} / 2$, at least for $V_{a}$ sufficiently lower than $V_{0}$. We furthermore find that the plasma density at the sheath edge is $n_{0} \mathrm{e}^{-1 / 2}$, which is also a classical result. $^{27}$

For $V_{a}>V_{0}$, saturating (15) we get:

$$
U_{e s h}=1+\frac{T_{i}}{T_{e}}\left(1-U_{i \mathrm{sh}}^{\max }\right) .
$$

This also leads to $n_{\mathrm{sh}}=n_{0} \mathrm{e}^{-1 / 2}$, and the potential at the sheath edge becomes

$$
V_{\mathrm{sh}}=V_{0}+\frac{T_{i}}{2}\left(1-U_{i \mathrm{sh}}^{\max }\right),
$$

with

$$
U_{i \mathrm{sh}}^{\max }=\mathrm{e}^{-\frac{2\left(V_{a}-V_{0}\right)}{T_{i}}} .
$$

\section{B. IV characteristics in the limit of big radius probes, and comparison with a classical model}

The $I V$ characteristic according to the big radius probe approximation is shown on figure 13 in comparison with the $I V$ curves calculated for cylindrical probes with different $R_{p}$, which resolves the apparent contradiction noted in Section III A regarding the discrepancy of the 1D Cartesian solution in Fig. 10.

The predictions of this model differ significantly from those of conventional theory, ${ }^{28}$ as illustrated on Fig. 14(a). The main reason for these differences arises from the modification of the electron current expression in the two models:

$$
\begin{gathered}
J_{e}^{\text {classical }}=q \frac{n_{0}}{4} \mathrm{e}^{\left(\frac{V_{a}-V_{0}}{T_{e}}\right)} \sqrt{\frac{8 q T_{e}}{\pi m_{e}}}, \\
J_{e}^{\text {big radius }}=q n_{0} \mathrm{e}^{-\frac{1}{2}} \mathrm{e}^{\left(\frac{V_{a}-V_{0}}{T_{e}}\right)} \sqrt{\frac{q T_{e}}{m_{e}}},
\end{gathered}
$$

for $V_{a}<V_{0}$, as shown in Fig. 14(b).

The ion current contributions for both models are also represented on figure 14(b). In classical theory, the ion current is taken to be constant for any value of the applied potential, whereas the big probe solution implies a continuously decreasing ion current as $V_{a}$ approaches, and passes, the plasma potential. This being said, the electron contribution to the total current is much higher than the ion current in this region, so the approximation of a constant ion flux is fairly reasonable. With regard to the ion saturation currents (figure 14(c)), the expressions for the two models can be compared as follows:

$$
\begin{gathered}
J_{i \mathrm{sat}}^{\text {classical }}=-q n_{0} \mathrm{e}^{-\frac{1}{2}} \sqrt{q T_{e} / m_{i}}, \\
J_{i \mathrm{sat}}^{\text {big radius }}=-q n_{0} \mathrm{e}^{-\frac{1}{2}} \sqrt{q\left(T_{e}+T_{i}\right) / m_{i}},
\end{gathered}
$$

where the big radius probe expression accounts for nonzero ion temperature.
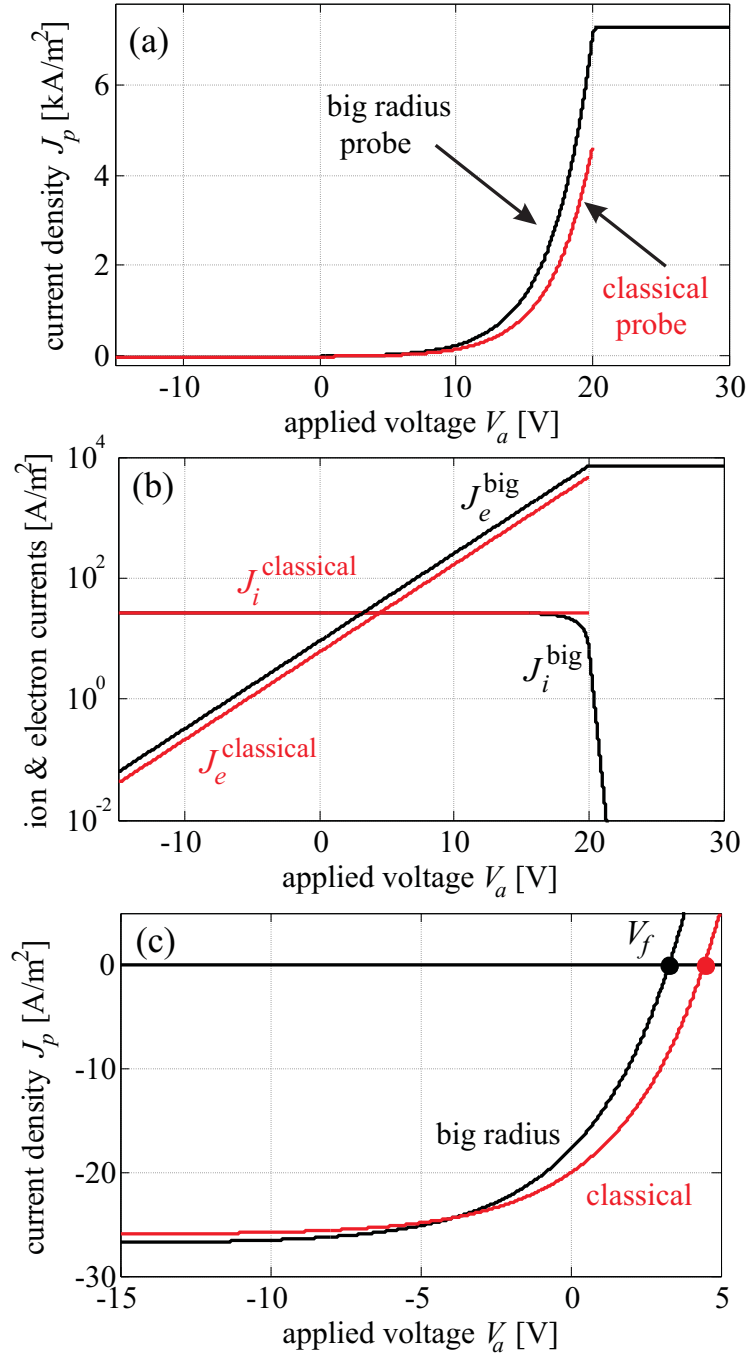

FIG. 14. (a) $I V$ characteristics for a classical model (red lines) compared with the big radius probe approximation (black lines); (b) semi-log plot of the electron currents, $J_{e}$, and magnitude of the ion currents, $J_{i}$; (c) expanded scale to show the ion saturation currents and the floating potentials $V_{f}$. Fixed parameters: Argon gas, $n_{0}=10^{17} \mathrm{~m}^{-3}, T_{i}=0.2 \mathrm{~V}, T_{e}=3 \mathrm{~V}$, $V_{0}=20 \mathrm{~V}$.

The modification of electron and ion flux expressions naturally implies different values for the floating potential:

$$
\begin{gathered}
V_{f}^{\text {classical }}=V_{0}-\frac{T_{e}}{2}\left[\ln \left(\frac{m_{i}}{2 \pi m_{e}}\right)+1\right]=V_{0}-\frac{T_{e}}{2} \ln \frac{\mathrm{e}^{1} m_{i}}{2 \pi m_{e}}, \\
V_{f}^{\text {big radius }}=V_{0}-\frac{T_{e}}{2} \ln \frac{T_{e}\left(m_{i}+m_{e}\right)}{m_{e}\left(T_{e}+T_{i}\right)} \cong V_{0}-\frac{T_{e}}{2} \ln \frac{m_{i}}{m_{e}},
\end{gathered}
$$

which differ by $\frac{T_{e}}{2} \ln \left(\frac{\mathrm{e}^{1}}{2 \pi}\right) \cong 0.4 T_{e}$ as shown in Fig. 14(c).

Finally, Fig. 15 shows the $I V$ characteristic predicted in the framework of the big probe approximation for the special case $T_{e}=T_{i}$, in order to compare with the numerical result of Parrot et al, ${ }^{36}$ also obtained in 


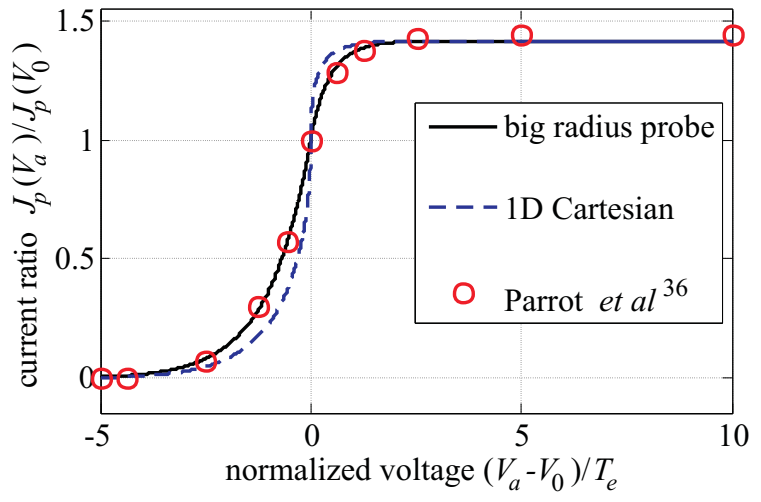

FIG. 15. Normalized current-voltage characteristics for the big radius probe, the $1 \mathrm{D}$ Cartesian case, and the numerical simulation of Parrot et $a l^{36}$ for the special case of $T_{i}=T_{e}$ and ionized oxygen atoms. $n_{0}=10^{17} \mathrm{~m}^{-3}, T_{i}=T_{e}=3 \mathrm{~V}$.

the limit of probe radius much larger than the sheath thickness. The correspondence between both curves is excellent and suggests a convergence of both approaches in the big radius probe limit, similarly to Fig. 11. Fig. 15 also shows the $I V$ characteristic for the $1 \mathrm{D}$ Cartesian infinite plane wall, which differs from the big radius probe limit due to their different boundary conditions at infinity.

In conclusion, it has been shown that the classical 1D Cartesian model of a collisionless sheath applies only in the limit of large radius cylindrical (or spherical) probes. This is why non-saturation effects observed experimentally for small probes could not be described by the classical sheath model. The big radius probe approximation relies on the supposition that the probe radius is much bigger than the typical length scale of the sheath, or plasma perturbation. We note that, with our set of parameters, this typical length scale is about $1 \mathrm{~mm}$, and therefore a "big probe" would in reality be several centimeters in diameter, and would rather be qualified as a big body.

\section{DISCUSSION}

\section{A. Concerning the extension of solutions up to infinity}

Clearly, whichever geometry is considered, an infinite plasma has no meaning if ionization is included, because an infinite plasma would generate infinite particle fluxes that would have to be adsorbed by the wall or the immersed body. In such models, a probe must be taken to be immersed in plasma of limited extent, or in other words, enclosed in a vessel. If the probe is small enough to be non-perturbing, the majority of the ionization contributes to fluxes toward external walls. Considering a simplified axisymmetric system in Fig. 16, the flux for a given species is oriented toward the probe in its vicin-

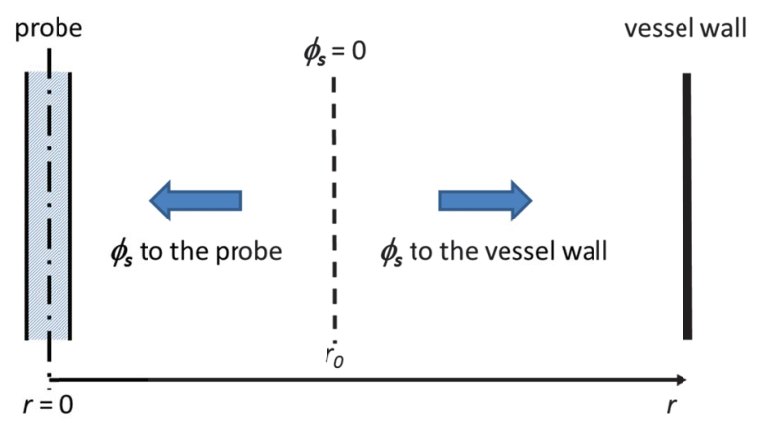

FIG. 16. Schematic of flows to a cylindrical probe and to a cylindrical wall, showing the watershed radius $r_{0}$.

ity while it is oriented toward the vessel wall far away from the probe. There is therefore a certain watershed radius $r_{0}$ at which the particle flux must vanish. ${ }^{3}$ The flux adsorbed by the probe is then given by the integral of all ionizations between the probe radius $R_{p}$ and $r_{0}$. For a given flux to the probe, decreasing the ionization rate leads to increasing $r_{0}$, which tends to infinity in the collisionless (ionizationless) limit. As a consequence, the extension of the perturbed region for cylindrical and spherical probes to infinity seems in fact to be required in the framework of collisionless models.

Then, consistent with the collisionless hypothesis, our solution extends from the probe to infinity, without any distinction possible between sheath and pre-sheath because the charge separation evolves continuously over the whole domain. This being said, although the solution is mathematically valid over the whole space, it is not physically valid everywhere: If the collisionless hypothesis is acceptable for low pressure in the vicinity of the probe (i.e. in the "sheath" where the charge separation is significant), over larger distances, collisions are determinant and can no longer be neglected. It should be noted, however, that there is a fundamental difference between 1D Cartesian and radial geometries: In the 1D Cartesian case, a collisional (ionization) presheath is required to justify the flux at the sheath entrance, ${ }^{6}$ while for radial probes, the inward flux concentration in the geometric presheath allows a collisionless presheath to relax asymptotically to the unperturbed plasma. ${ }^{11,12}$

\section{B. Concerning the repelled species convective velocity at the probe surface}

In the 1D Cartesian demonstration it was shown that the repelled species, $s_{r}$, has a convective velocity at the probe surface, $u_{s_{r} a}$, which is undetermined but must lie in an interval $\left[0, u_{\mathrm{th} s_{r}}\right]$. It was further shown that, in the field free situation, $u_{\mathrm{th} s_{r}}$ is the only possible value for $u_{s_{r} a}$ in order to ensure continuous solutions when the bias of the wall crosses the plasma potential. We then extended this result to all bias potentials for the repelled species. By doing so we actually converge with the con- 
ventional approach in which the electron flux at the wall surface is taken to be given by the random thermal kinetic flux ${ }^{1-3,8,9,13,19,26-28,32,43,44} \frac{1}{4} n_{e a} \bar{v}_{e}=\frac{1}{\sqrt{2 \pi}} n_{e a} u_{\text {the }}$, at least, for the case $V_{a}<V_{0}$. The only difference is the factor $\frac{1}{\sqrt{2 \pi}}$ which does not appear in our solution. The classical kinetic theory flux, $\frac{1}{4} n_{e a} \sqrt{\frac{8 q T_{e}}{\pi m_{e}}}$, applies to the case of non-interacting particles in front of an adsorbing wall: In a kinetic description, the velocity distribution is truncated because no particles return from the wall. The classical kinetic theory result thus corresponds to the flux of a half-Maxwellian velocity distribution. However, in the fluid approach considered here, Maxwellian velocity distributions are assumed to persist up to the adsorbing wall surface. Our solution then self-consistently demonstrates that the predicted net flux convected to the wall, $n_{e a} \sqrt{\frac{q T_{e}}{m_{e}}}$, is larger than the classical kinetic theory result by a factor $\sqrt{2 \pi}$.

In the cylindrical and spherical $1 \mathrm{D}$ radial cases the repelled species velocity at the probe is also found to lie in the interval $\left[0, u_{\mathrm{th} s_{r}}\right]$, but no condition was found for the field free situation. On the contrary, we have demonstrated the existence of a zone in terms of probe bias, in the vicinity of the plasma potential $V_{0}$, in which the convective velocities of both repelled and field accelerated species could fall below $u_{\text {th }}$. Our main question here concerns the application of 1D Cartesian results to cylindrical and spherical 1D radial cases. In fact, to solve the $1 \mathrm{D}$ radial problem we postulated that the field-free convective velocity at the probe for the repelled species is also $u_{\mathrm{th} s_{r}}$, as it must be in the $1 \mathrm{D}$ Cartesian situation. By doing so we have in fact made the conventional assumption that the adsorption mechanism of the repelled species at the biased surface does not depend on the geometry considered.

\section{The criterion on initial velocities}

According to our demonstration, a criterion on initial velocities seems to have no meaning apart from the $1 \mathrm{D}$ Cartesian model for which the flux conservation imposes non-vanishing velocities at infinity, or at the sheath edge in the framework of the big radius probe approximation. In 1D Cartesian systems, the criterion on initial velocities arises from the requirement of a continuouslydecreasing charge density when going away from the wall, up to infinity or to the sheath edge where electrons and ions have the same density. In 1D radial geometries, all the Lambert function arguments vanish in the limit of large $r$, and the requirement of a continuously-decreasing charge density up to infinity leads to the trivial condition $1 / T_{e}+1 / T_{i} \geq 0$. In this case, another criterion is required to determine the charge separation (or, equivalently, the electric potential) at the sonic radius.

\section{CONCLUSIONS}

The two-fluid equations were solved using the Lambert function for the 1D cases of planar, cylindrical and spherical Langmuir probes in a steady state, isothermal plasma. Because electron inertia and ion temperature are both included, the fluid equations are symmetric in the terms for electrons and ions, hence the solutions apply below and above the plasma potential. The solution of the fluid equations allows ion and electron densities to be expressed as functions of the electric potential, which is then the only remaining function implied in Poisson's equation. This final differential equation is integrated numerically to obtain the full solution, i.e. the spatial dependence of all parameters. The calculated current-voltage characteristics for radial probes correspond with general experimental observations, such as non-saturation of the ion and electron saturation currents, which are more pronounced for small probe radii. Furthermore, spherical probe currents show stronger non-saturation than cylindrical probes for identical radius.

The model gives a single $1 \mathrm{D}$ solution for radial probes extending from the probe surface up to infinity. The connection with classical models considering a separation of space between a 1D Cartesian collisionless sheath and a quasineutral pre-sheath was made, and it was demonstrated that this approach is valid only as an approximation in the framework of big radius probes for which the sheath length scale can be considered to be very small compared to the probe radius. In the limit of big radius probes, the solution leads to several generalized results which converge towards conventional ones provided that proper approximations are applied. Notably, a criterion on initial velocities was derived, generalizing the Bohm criterion, which not only implies a minimal initial velocity for ions entering an ion-rich sheath, but also for electrons when the probe bias is higher than the plasma potential. This unique expression for both species was shown to be related to the total required kinetic energy density of ions and electrons at the sheath entrance. Saturation of the criterion minimizes this energy density, as well as the total charge of the perturbed region; this case then corresponds to the minimum perturbation to the plasma by the probe for any given probe potential.

Another surprising result of the 1D Cartesian solution is the prediction, in the field free situation, of an adsorbed particle flux by the wall that differs from the usually-stated thermal kinetic flux by a factor $\frac{1}{\sqrt{2 \pi}}$. This arises from the requirement of continuous solutions when the wall bias potential crosses the plasma potential. As nothing distinguishes a charged particle from a neutral one in the field free situation, this result can in principle be generalized to neutral particle fluxes at an adsorbing surface. 


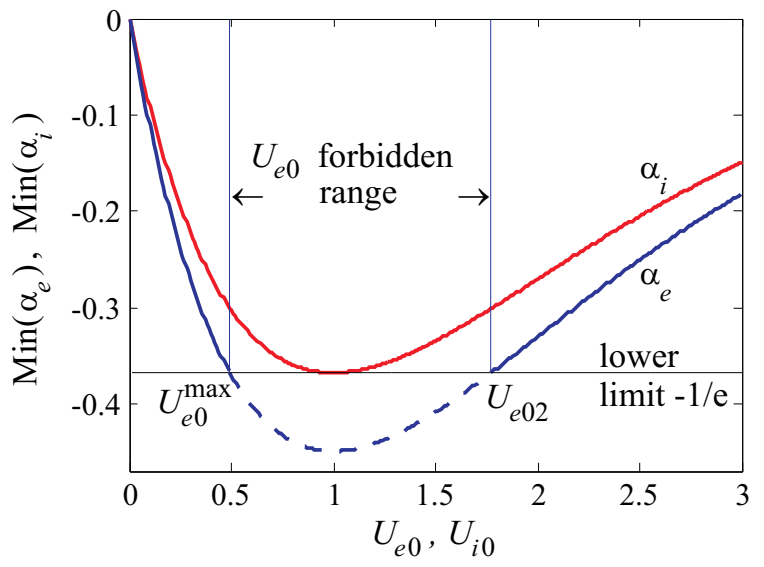

FIG. 17. Minimum values of the Lambert function arguments $\alpha_{e}\left(U_{e 0}, V_{a}\right)$ as a function of $U_{e 0}$ (blue curve); and $\alpha_{i}\left(U_{i 0}, V_{0}\right)$ as a function of $U_{i 0}$ (red curve), for the case $V_{a} \leq V_{0}$. Nontypical parameters are used for clearer presentation: $V_{0}=$ $20 \mathrm{~V}, V_{a}=19.9 \mathrm{~V}, T_{e}=1 \mathrm{~V}$, and $T_{i}=0.2 \mathrm{~V}$.

\section{Appendix A: 1D Cartesian solution}

The solutions (10) must be real and positive, which implies that the Lambert functions must be real and negative. The reality condition implies that $U_{e, i}$ must be described by the $W_{0}$ and $W_{-1}$ branches of Lambert function, and negative values are obtained only provided that the arguments $\alpha_{e, i}$ are restricted to the interval $\left[-\frac{1}{\mathrm{e}}, 0\right]$. We therefore write:

$$
\begin{gathered}
-\frac{1}{\mathrm{e}} \leq-U_{e 0} \mathrm{e}^{-U_{e 0}-\frac{2\left(V-V_{0}\right)}{T_{e}}}=\alpha_{e}\left(U_{e 0}, V\right) \leq 0 \\
-\frac{1}{\mathrm{e}} \leq-U_{i 0} \mathrm{e}^{-U_{i 0}+\frac{2\left(V-V_{0}\right)}{T_{i}}}=\alpha_{i}\left(U_{i 0}, V\right) \leq 0
\end{gathered}
$$

The upper limit is always satisfied and we have only to deal with the lower one. These inequalities must be satisfied for all space, from infinity where $V=V_{0}$, to the wall where a potential $V_{a}$ is applied. The solutions depend on whether the probe applied potential $V_{a}$ is less than, greater than, or equal to the plasma potential $V_{0}$. We consider here the case $V_{a} \leq V_{0}$, the reasoning for the case $V_{a} \geq V_{0}$ being similar with inverted roles for ions and electrons, accounting for charge sign.

The potential $V$ is assumed to vary monotonically with $x$ for any applied potential $V_{a}$. Under this condition, for $V_{a} \leq V_{0}$, the argument $\alpha_{e}\left(U_{e 0}, V\right)$ reaches its minimum at the wall where $V=V_{a}$, for any value of $U_{e 0}$. On the other hand, for any $U_{i 0}$, the minimum value for $\alpha_{i}\left(U_{i 0}, V\right)$ is reached at infinity when $V \rightarrow V_{0}$. It then appears that the inequalities (A1) in fact only concern the boundary conditions at the wall and infinity. Plots of $\alpha_{e}\left(U_{e 0}, V_{a}\right)$ and $\alpha_{i}\left(U_{i 0}, V_{0}\right)$ for these two extreme cases are shown in figure 17.

For ions, (A1) is satisfied for any value of $U_{i 0}$ and then no condition on the initial velocity arises in this
$V_{a} \leq V_{0}$ case. The potential $V(x)$ decreases monotonically when approaching the wall surface, and so $\alpha_{i}$ increases. The first branch of Lambert functions, $W_{0}(\alpha)$, tends monotonically toward 0 for increasing argument, while the second branch, $W_{-1}(\alpha)$, tends towards infinity (Fig. 2). Then, if the ion square velocity $U_{i}$ were to be represented by the $W_{0}$ branch, it would imply a deceleration of ions when approaching the wall surface. However, the ion velocity is expected to increase, and therefore the $W_{-1}$ branch must apply for the ion solution for the case $V_{a} \leq V_{0}$, and so $U_{i 0} \geq 1$.

Regarding electrons, the argument $\alpha_{e}\left(U_{e 0}, V_{a}\right)$ always crosses the lower limit $-\frac{1}{\mathrm{e}}$ at two points in Fig. 17, whose values for $U_{e 0}$ are also given by Lambert functions using (A1):

$$
\begin{aligned}
& U_{e 0}^{\max }=-W_{0}\left[-\mathrm{e}^{\frac{2\left(V_{a}-V_{0}\right)}{T_{e}}-1}\right] \leq 1, \\
& U_{e 02}=-W_{-1}\left[-\mathrm{e}^{\frac{2\left(V_{a}-V_{0}\right)}{T_{e}}-1}\right] \geq 1 .
\end{aligned}
$$

For both points the electron square normalized velocity at the wall takes the value $U_{e}=1$, i.e. $u_{e}=u_{\text {the }}$ at the wall, since $\alpha_{e}=-\frac{1}{\mathrm{e}}$ in (10). The solutions with $U_{e 0} \geq U_{e 02}$ correspond to initial velocities $u_{e 0}$ greater than $u_{\text {the }}$ and would be described by the $W_{-1}$ branch of the Lambert function. According to these solutions, the electrons would come from infinity with a "high" convective velocity and would be decelerated as they approach the wall. However, considering particle flux conservation, if the convective velocity of electrons was to decrease, their density should on the contrary increase when approaching the wall to maintain the constant flux. As we expect the opposite to occur, these solutions can be eliminated. On the other hand, solutions for $U_{e 0} \leq U_{e 0}^{\max } \leq 1$ correspond to initial velocities lower than $u_{\text {the }}$ and are described by the $W_{0}$ branch, for which electrons are correctly predicted to be accelerated towards the wall. Then, in this $V_{a}<V_{0}$ case the solutions to (10) are given by (11) and (12).

\section{Appendix B: 1D cylindrical solution}

\section{Around the ion sonic radius $R_{S}$ :}

A mathematical difficulty arises at the sonic radius regarding density and velocity gradients for the field accelerated species, because both numerator and denominator in the derivative of (30) and (37) vanish at $r=R_{S}$. This indeterminacy can be overcome by using a Taylor expansion for the electric potential around $R_{S}$ and performing a power series expansion ${ }^{29}$ of velocity and density expressions around $R_{S}$. As an example we show here the development for the $V_{a} \leq V_{0}$ case.

We define the net charge density at $r=R_{S}$ to be:

$$
Q_{S}=q\left(n_{i}\left(R_{S}\right)-n_{e}\left(R_{S}\right)\right)
$$




$$
=q n_{0}\left[\mathrm{e}^{-\frac{1}{2}-\frac{V_{S}-V_{0}}{T_{i}}}-\mathrm{e}^{\frac{1}{2} W_{0}\left[-\frac{R_{p}^{2}}{R_{S}^{2}} \mathrm{e}^{\frac{2\left(V_{a}-V_{S}\right)}{T_{e}}-1}\right]+\frac{V_{S}-V_{0}}{T_{e}}}(\mathbf{B} 1)\right.
$$

Then from (38) and (39) we get:

$$
\left[\partial_{r}^{2} V\right]_{R_{S}}=-\frac{Q_{S}}{\varepsilon_{0}}-\frac{T_{i}}{R_{S}^{2}}
$$

According to (38) and (B2), a second order Taylor expansion of the potential around $R_{S}$ can be expressed as:

$$
V=V_{S}+\frac{T_{i}}{R_{S}} \epsilon-\frac{1}{2}\left(\frac{Q_{S}}{\varepsilon_{0}}+\frac{T_{i}}{R_{S}^{2}}\right) \epsilon^{2},
$$

with $\epsilon=r-R_{S}$. Using (B3), the Taylor expansions of $U_{i}$ and $n_{i}$ around $R_{S}$ can be expressed, from which the first derivatives at the sonic radius are identified to be:

$$
\begin{array}{r}
{\left[\partial_{r} U_{i}\right]_{R_{S}}=-\sqrt{\frac{2 Q_{S}}{\varepsilon_{0} T_{i}}},} \\
{\left[\partial_{r} n_{i}\right]_{R_{S}}=n_{0} \mathrm{e}^{-\frac{1}{2}+\frac{V_{0}-V_{S}}{T_{i}}}\left(\sqrt{\frac{Q_{S}}{2 \varepsilon_{0} T_{i}}}-\frac{1}{R_{S}}\right) .}
\end{array}
$$

It then appears that the charge separation $Q_{S}$ gives directly the gradient of the ion square velocity at the sonic radius. We also note from (B4) that the charge separation at $R_{S}$ must be positive, $n_{i}\left(R_{S}\right) \geq n_{e}\left(R_{S}\right)$, for $\left[\partial_{r} U_{i}\right]_{R_{S}}$ and $\left[\partial_{r} n_{i}\right]_{R_{S}}$ to be real numbers, as expected for $V_{a} \leq V_{0}$. Furthermore, as in most cases we expect the ion density to increase with $r$, we can express from (B4) the condition:

$$
\left[\partial_{r} n_{i}\right]_{R_{S}} \geq 0 \Rightarrow Q_{S} \geq \frac{2 \varepsilon_{0} T_{i}}{R_{S}^{2}} .
$$

The inequality (B5) defines an upper value for $V_{S}$ but can unfortunately not be solved analytically.

In the $V_{a} \geq V_{0}$ case the density and velocity gradients for electrons at the sonic radius are given by:

$$
\begin{array}{r}
{\left[\partial_{r} U_{e}\right]_{R_{S}}=-\sqrt{\frac{-2 Q_{S}}{\varepsilon_{0} T_{e}}},} \\
{\left[\partial_{r} n_{e}\right]_{R_{S}}=n_{0} \mathrm{e}^{-\frac{1}{2}-\frac{V_{0}-V_{S}}{T_{e}}}\left(\sqrt{\frac{-Q_{S}}{2 \varepsilon_{0} T_{e}}}-\frac{1}{R_{S}}\right) .}
\end{array}
$$

As expected the charge separation is now to be negative in order to obtain real quantities. The electron density is expected to increase with $r$, such as we can express from (B6) the following condition on the charge separation at the sonic radius in the $V_{a} \geq V_{0}$ case:

$$
\left[\partial_{r} n_{e}\right]_{R_{S}} \geq 0 \Rightarrow Q_{S} \leq-\frac{2 \varepsilon_{0} T_{e}}{R_{S}^{2}} .
$$

Coming back to the general case, the range of possible values for $V_{S}$ can be analytically limited by considering

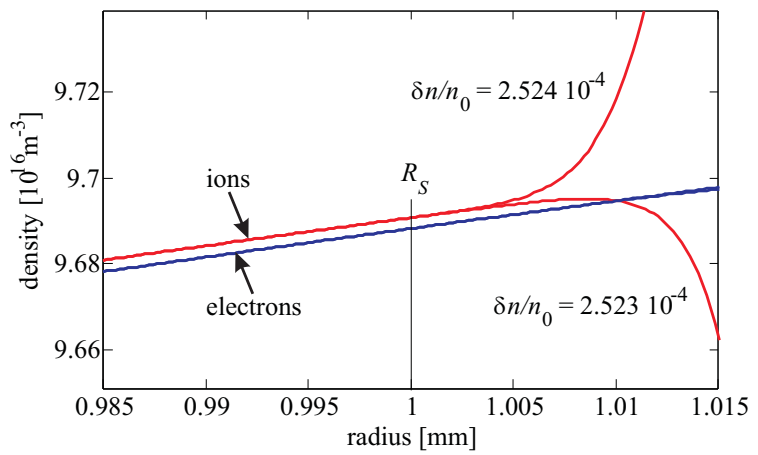

FIG. 18. Electron and ion densities around the ion sonic radius $R_{S}$ obtained by integration of Poisson's equation for two close values of $Q_{S}(0.04 \%$ variation $)$. Fixed parameters: Argon gas, $n_{0}=10^{17} \mathrm{~m}^{-3}, T_{e}=3 \mathrm{~V}, T_{i}=0.2 \mathrm{~V}, V_{0}=20 \mathrm{~V}$, $V_{a}=15 \mathrm{~V}$, probe radius $R_{p}=0.25 \mathrm{~mm}$.

first that the density of the field accelerated species at the sonic radius cannot exceed its initial density at infinity $n_{0}$, and secondly that the density of the field repelled species at the sonic radius must be smaller than that of the field accelerated one $\left(Q_{S} \geq 0\right.$ for $V_{a} \leq V_{0}$ and $Q_{S} \leq 0$ for $\left.V_{a} \geq V_{0}\right)$.

Considering the case $V_{a} \leq V_{0}$ we get:

$$
V_{0}-\frac{T_{i}}{2} \leq V_{S} \leq V_{0}-\frac{\Theta}{2}-\frac{T_{i}}{2} W_{0}\left[-\frac{\Theta R_{p}^{2}}{T_{i} R_{s}^{2}} \mathrm{e}^{\frac{2\left(V_{a}-V_{0}\right)}{T_{e}}-\frac{\Theta}{T_{i}}}\right]
$$

where $\Theta=\frac{T_{e} T_{i}}{T_{e}+T_{i}}$.

For $V_{a} \geq V_{0}$ the range for $V_{S}$ is given by:

$$
V_{0}+\frac{T_{e}}{2} \geq V_{S} \geq V_{0}+\frac{\Theta}{2}+\frac{T_{e}}{2} W_{0}\left[-\frac{\Theta R_{p}^{2}}{T_{e} R_{s}^{2}} \mathrm{e}^{\frac{-2\left(V_{a}-V_{0}\right)}{T_{i}}-\frac{\Theta}{T_{e}}}\right]
$$

\section{Iterative integrations}

For a given $R_{S}$ the result of Poisson integration in the direction of increasing $r$ is extremely sensitive to the value taken for $Q_{S}$ (i.e. $V_{S}$ through (B1)). This is illustrated for a $V_{a} \leq V_{0}$ case on figure 18 which represents the evolution of the electron and ion densities for two very close values of $Q_{S}(0.04 \%$ variation). A too high charge separation at $R_{S}$ (i.e. velocity and density gradients too steep (B4)) leads to an ion density which diverges toward $+\infty$, while, on the other hand, a too low charge separation leads to an ion density which diverges toward $-\infty$. The divergences occur especially when the space charge density $Q$ becomes small, and are then so sensitive that one can try to adjust the value of $Q_{S}$ up to many decimal places without really improving the obtained density profiles. Nevertheless, $Q_{S}$ can then be iteratively determined by detecting this positive/negative divergence switching. Apart from this numerical determination, we would need a criterion on $Q_{S}$ that would ensure $V(r \rightarrow \infty)=V_{0}$. Such a criterion cannot be deduced for the cylindrical 

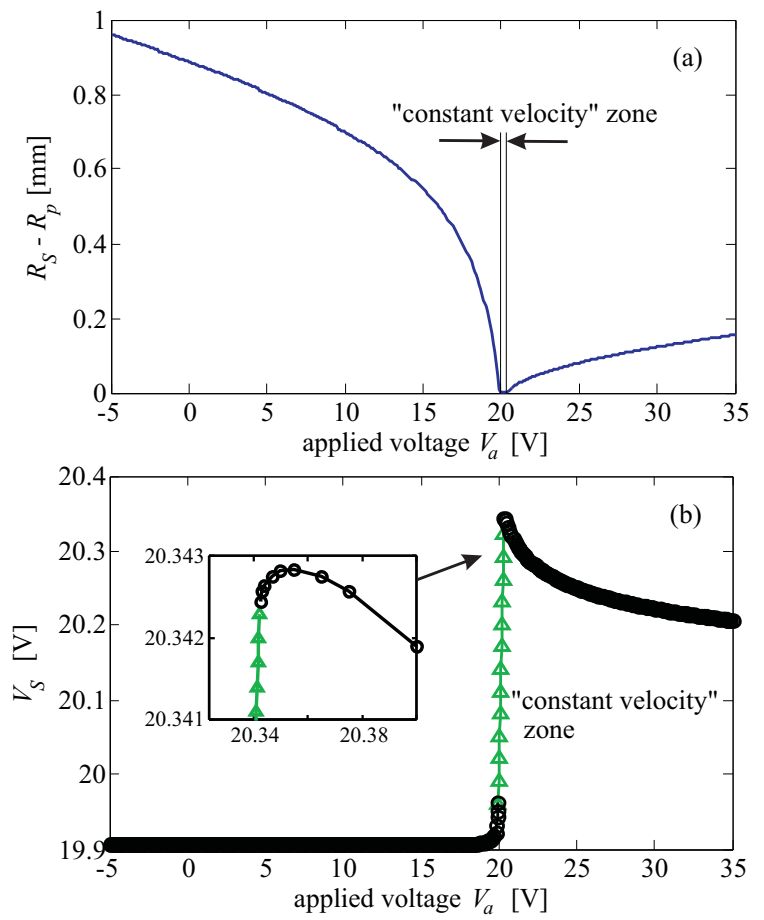

FIG. 19. (a) Evolution of the sonic radius $R_{S}$ with probe bias potential $V_{a}$, for probe radius $R_{p}=0.15 \mathrm{~mm}$. The "constant velocity" zone extends approximately between $V_{\lim }=$ $19.955 \mathrm{~V}<V_{0}$ and $V_{\lim }=20.342 \mathrm{~V}>V_{0}$. (b) Evolution of the electric potential $V_{S}$ at the sonic radius with $V_{a}$. The triangles represent the "constant velocity" zone solution $V_{S}\left(V_{a}\right)=V_{a}$, while the circles correspond to computed solutions outside of the "constant velocity" zone. The inset shows a zoom around the limit potential for $V_{a} \geq V_{0}$. Fixed parameters: Argon gas, $n_{0}=10^{17} \mathrm{~m}^{-3}, T_{i}=0.2 \mathrm{~V}, T_{e}=3 \mathrm{~V}, V_{0}=20 \mathrm{~V}$.

case in the same way as for the 1D Cartesian case (using $\left.\left[\partial_{V}|Q|\right]_{V_{0}} \leq 0\right)$ because all the expressions vanish at infinity. Fortunately, the integration of Poisson's equation towards the probe (decreasing $r$ ) is not so sensitive to the value taken for $Q_{S}$, and only a few iterations are needed to estimate the voltage profile between $R_{S}$ and $R_{p}$ with a sufficient resolution (1\% in Fig. 9).

\section{Voltage dependence of the sonic radius parameters}

The typical evolution $\left(R_{p}=0.15 \mathrm{~mm}\right)$ of the sonic radius $R_{S}$ with the applied probe potential $V_{a}$ is shown in Fig. 19(a). It can be seen that the "constant velocity" zone for which $R_{S}=R_{p}$, and both electron and ion velocities are taken to be constant $\left(u_{s}=u_{\text {ths }}\right)$, is of small extent. It was found that the width of the "constant velocity" zone depends on the probe radius, lower $R_{p}$ leading to wider zones. The increase of $R_{S}$ with the applied potential was found to be the only reason for the non-saturation of the ion and electron currents. To illustrate this point we consider as an example the electron current non-saturation for $V_{a}>V_{0}$. In this case, the

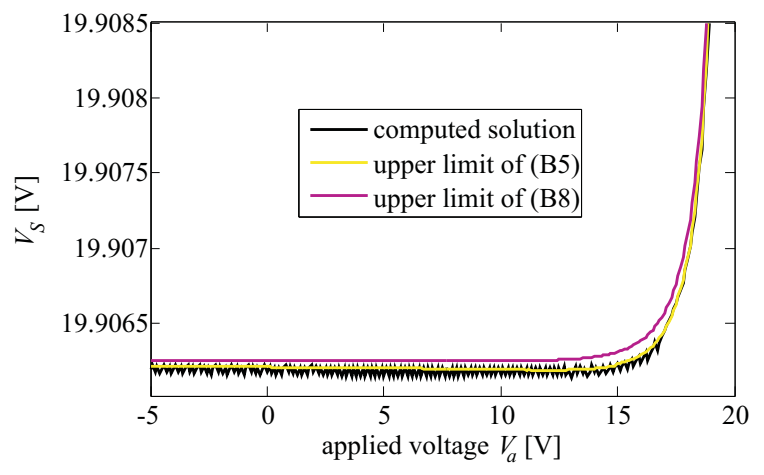

FIG. 20. Evolution of the electric potential $V_{S}$ as a function of the applied probe potential $V_{a} \leq V_{0}$, for $R_{p}=0.15 \mathrm{~mm}$. The noisy black curve corresponds to computed values. The yellow curve is the solution of (B5) while the red one corresponds to the analytical upper value for $V_{S}$ according to (B8). Fixed parameters: Argon gas, $n_{0}=10^{17} \mathrm{~m}^{-3}, T_{i}=0.2 \mathrm{~V}, T_{e}=3 \mathrm{~V}$, $V_{0}=20 \mathrm{~V}$.

ion contribution to the total current can be neglected, so that the electron flux to the probe $\Gamma_{e}=2 \pi R_{S} u_{\text {the }} n_{e S}$ determines the collected current. It can been verified (not shown) that $n_{e S}$ decreases slightly (by a few percent) with increasing bias, and therefore the non-saturation of the electron flux can only be due to the increase in $R_{S}$ with $V_{a}$. Similar reasoning can be applied for the nonsaturation of ion current.

Figure 19(b) shows the evolution of the sonic radius potential $V_{S}$ with the probe potential. We first note that the solution computed in the $V_{a}$ ranges for which $R_{S}$ can be defined seems to tend tangentially toward the "constant velocity" zone solution $V_{S}\left(V_{a}\right)=V_{a}$ at both limit potentials $V_{\text {lim. }}$. Fig. 20 shows a zoom on $V_{S}\left(V_{a}\right)$ for the $V_{a} \leq V_{0}$ range, along with the analytic upper limit for $V_{S}$ given by (B8), which corresponds to a vanishing charge separation at the sonic radius. The computed upper limit given by (B5) expresses the condition for vanishing ion density gradient at $R_{S}$. The fact that the computed values of $V_{S}$ deviate only slightly from these limit curves is a consequence of the very small charge separation at the sonic radius for $V_{a} \leq V_{0}$. This then cannot be generalized to the $V_{a} \geq V_{0}$ case for which it was shown that the charge separation at $R_{S}$ is large.

\section{Analysis of cylindrical probe characteristics}

We describe here how the cylindrical solution given in this work can be used to analyse experimental $I V$ characteristics in a simple way. To illustrate the method we consider as an example a theoretical characteristic $J_{p}\left(V_{a}\right)$ computed for $R_{p}=0.15 \mathrm{~mm}$, taken from Fig. 10, as shown on Fig. 21(a).

First, radial probes characteristics are generally expected to present an inflexion point in the vicinity of 

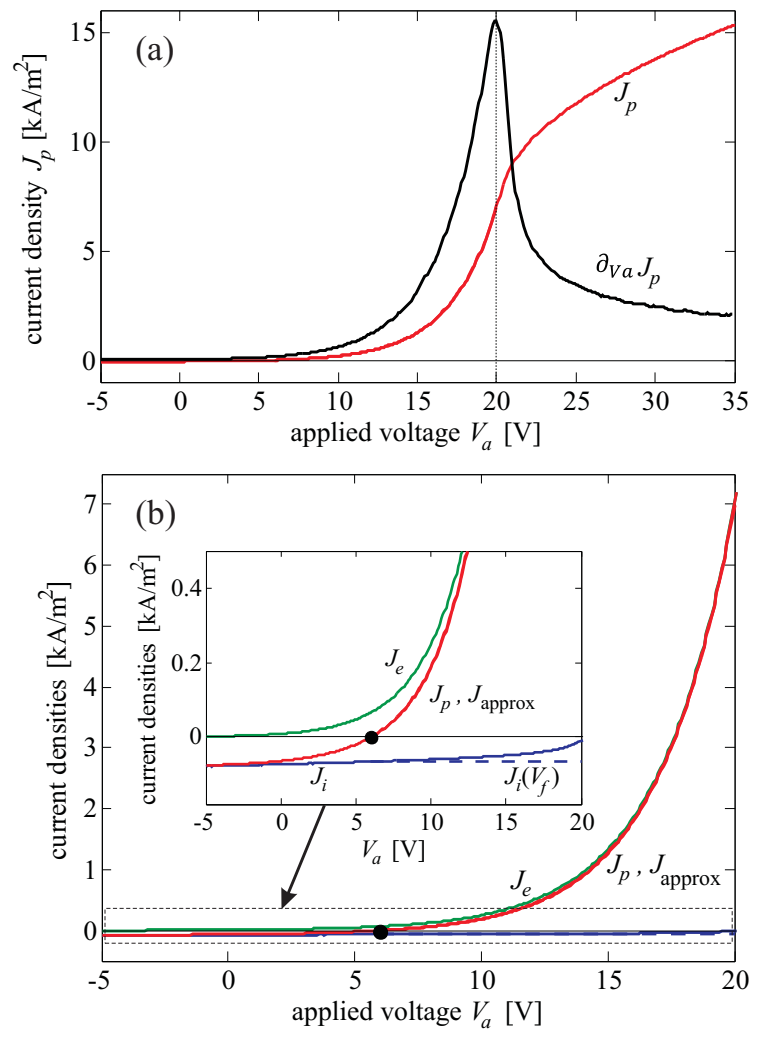

FIG. 21. (a) $I V$ characteristic calculated for $R_{p}=0.15 \mathrm{~mm}$, with the first derivative $\partial_{V_{a}} J_{p}\left(V_{a}\right)$, whose maximum indicates the plasma potential. (b) The probe current $J_{p}$ and the approximated current $J_{\text {approx }}$, which are indistinguishable in these graphs, and the electron current $J_{e}$; the inset shows an expanded scale of the currents around the floating potential $V_{f}$ (marked by a black dot at $J_{p}=0$ ), along with the ion current $J_{i}$ and the constant ion current $J_{i}\left(V_{f}\right)$ (dashed line). Fixed parameters: Argon gas, $n_{0}=10^{17} \mathrm{~m}^{-3}, T_{i}=0.2 \mathrm{~V}$, $T_{e}=3 \mathrm{~V}, V_{0}=20 \mathrm{~V}$.

the plasma potential $V_{0}$. This is illustrated on Fig. 21(a) showing the first derivative $\partial_{V_{a}} J_{p}\left(V_{a}\right)$, which reaches its maximum slightly before $V_{a}=V_{0}$. Theoretically, for big radius probes this inflexion point is exactly at $V_{0}$. It is sometime difficult to determine precisely this point from real measurements, but it provides a fair order of magnitude. Another particular point of the characteristic is the floating potential $V_{f}$, for which ion and electron contributions to the total current are equal. We propose an analysis of the portion of the curve comprised between the floating potential and the plasma potential.

According to the model, electron and ion currents $\left(J_{e}\right.$ and $J_{i}$ respectively) at the probe surface are given by:

$$
\begin{array}{r}
J_{e}=q u_{\text {the }} n_{0} \mathrm{e}^{-\frac{1}{2}} \mathrm{e}^{\frac{V_{a}-V_{0}}{T_{e}}}, \\
J_{i}=-q u_{\text {th } i} n_{0} \sqrt{U_{i}\left(V_{a}\right)} \mathrm{e}^{-\frac{U_{i}\left(V_{a}\right)}{2}-\frac{V_{a}-V_{0}}{T_{i}}} .
\end{array}
$$

with

$$
U_{i}=-W_{-1}\left[-\frac{R_{S}^{2}}{R_{p}^{2}} \mathrm{e}^{\frac{-2\left(V_{S}-V_{a}\right)}{T_{i}}-1}\right] .
$$

Plots of $J_{e}$ and computed $J_{i}$ in the range $V_{a}=\left[V_{f}, V_{0}\right]$ are shown on figure 21(b). The relation (B11) is quite unpractical to use because both evolutions of $R_{S}$ and $V_{S}$ with the applied bias $V_{a}$ are unknown, and must be computed for a given probe radius $R_{p}$. But, as can be seen on the inset of figure 21(b), the ion contribution $J_{i}$ to the total current $J_{p}=J_{e}+J_{i}$ is rapidly negligible with respect to the electron one. In fact, we would make only a small error by considering the ion contribution to be constant between $V_{f}$ and $V_{0}$, this constant being given by the value of $J_{i}$ at the floating potential. According to equation B10 we have:

$$
J_{i}\left(V_{f}\right)=-J_{e}\left(V_{f}\right)=-q u_{\text {the }} n_{0} \mathrm{e}^{-\frac{1}{2}} \mathrm{e}^{\frac{V_{f}-V_{0}}{T_{e}}},
$$

therefore the total collected current $J\left(V_{a}\right)$ in the $\left[V_{f}, V_{0}\right]$ interval approximates to:

$$
\begin{aligned}
& J_{\text {approx }}\left(V_{a}\right)=J_{i}\left(V_{f}\right)+J_{e}\left(V_{a}\right) \\
= & q u_{\text {the }} n_{0} \mathrm{e}^{-\frac{1}{2}-\frac{V_{0}}{T_{e}}}\left[\mathrm{e}^{\frac{V_{a}}{T_{e}}}-\mathrm{e}^{\frac{V_{f}}{T_{e}}}\right] .
\end{aligned}
$$

We then obtain an expression which is independent of unknown parameters $R_{S}$ and $V_{S}$, and on the probe radius $R_{p}$ as well. The impact of $R_{p}$ on the characteristic is in fact taken into account through the experimental determination of the floating potential $V_{f}$, which depends on the probe radius as can be seen in (40). The difference between the originally-computed characteristic $J_{p}\left(V_{a}\right)$ and the approximate expression $J_{\text {approx }}\left(V_{a}\right)$ is so small as to be practically indistinguishable on Figs. 21(a) and (b) for $V_{a} \leq V_{0}$.

${ }^{1}$ F. F. Chen, Electric Probes (in "Plasma Diagnostic Techniques", ed. R. H. Huddlestone and S. L. Leonard, Academic Press, New York, 1965) Chap. 4, pp. 113-200.

${ }^{2}$ H. M. Mott-Smith and I. Langmuir, Phys. Rev. 28, 727 (1926).

${ }^{3}$ L. Tonks and I. Langmuir, Phys. Rev. 34, 876 (1929).

${ }^{4}$ J. E. Allen, Plasma Sources Sci. Technol. 18, 014004 (2009).

${ }^{5}$ D. Bohm, The Characteristics of Electrical Discharges in Magnetic Fields, edited by A. Guthrie and R. K. Wakerling (New York: McGraw-Hill, 1949) p. 77.

${ }^{6}$ A. Caruso and A. Cavaliere, Nuovo Cimento 26, 1389 (1962).

${ }^{7}$ S. A. Self, Phys. Fl. 6, 1762 (1963).

${ }^{8}$ S. H. Lam, Phys. Fl. 8, 73 (1965).

${ }^{9}$ R. N. Franklin and J. R. Ockendon, J. Plasma Phys. 4, 371 (1970).

${ }^{10}$ V. A. Godyak and N. Sternberg, IEEE Trans. Plasma Sci. 18, 159 (1990).

${ }^{11}$ K.-U. Riemann, J. Phys. D: Appl. Phys. 24, 493 (1991).

${ }^{12}$ K.-U. Riemann, J. Tech. Phys. 41, 89 (2000).

${ }^{13}$ R. N. Franklin and J. Snell, Phys. Pl. 8, 643 (2001).

${ }^{14}$ R. N. Franklin, J. Phys. D: Appl. Phys. 36, R309 (2003).

${ }^{15}$ K.-U. Riemann, Plasma Sources Sci. Technol. 18, 014006 (2009).

${ }^{16}$ K.-U. Riemann, Plasma Sources Sci. Technol. 18, 014007 (2009).

${ }^{17}$ R. M. Crespo, Plasma Sources Sci. Technol. 24, 045012 (2015).

${ }^{18}$ A. Din, Phys. Pl. 23, 083505 (2016).

${ }^{19}$ D. Bohm, E. H. S. Burhop, and H. S. W. Massey, The Characteristics of Electrical Discharges in Magnetic Fields, edited by A. Guthrie and R. K. Wakerling (New York: McGraw-Hill, 1949) Chap. 2, p. 13. 
${ }^{20}$ J. E. Allen, R. L. F. Boyd, and P. Reynolds, Proc. Phys. Soc. 70, 297 (1957).

${ }^{21}$ I. B. Bernstein and I. N. Rabinowitz, Phys. Fl. 2, 112 (1959).

${ }^{22}$ E. R. Harrison and W. B. Thompson, Proc. Phys. Soc. (London) 74, 145 (1959).

${ }^{23}$ K.-B. Persson, Phys. Fl. 5, 1625 (1962).

${ }^{24}$ P. D. Prewett and J. E. Allen, J. Plasma Phys. 10, 451 (1973).

${ }^{25}$ G. A. Emmert, R. M. Wieland, A. T. Mense, and J. N. Davidson, Phys. Fl. 23, 803 (1980).

${ }^{26}$ F. F. Chen, J. D. Evans, and D. Arnush, Phys. Pl. 9, 1449 (2002).

${ }^{27}$ I. H. Hutchinson, Principles of Plasma Diagnostics (Cambridge University Press, 2nd ed., 2002).

${ }^{28}$ M. A. Lieberman and A. J. Lichtenberg, Principles of Plasma Discharges and Materials Processing (John Wiley and Sons, 2nd ed., Hoboken, New Jersey, 2005).

${ }^{29}$ R. M. Corless, G. H. Gonnet, D. E. G. Hare, D. J. Jeffrey, and D. E. Knuth, Adv. Comput. Math. 5, 329 (1996).

${ }^{30}$ B. A. Alterkop, I. D. Dubinova, and A. E. Dubinov, J. Exp. Theor. Phys. 102, 173 (2006).

${ }^{31}$ B. Scheiner, S. D. Baalrud, B. T. Yee, M. M. Hopkins, and E. V. Barnat, Phys. Pl. 22, 123520 (2015).

${ }^{32}$ J. G. Laframboise, Univ. of Toronto, Institute for Aerospace Studies, UTIAS Report No. 100 (unpublished, 1966).

${ }^{33}$ R. N. Franklin and J. Snell, Phys. Pl. 7, 3077 (2000).

${ }^{34}$ V. Pletnev and J. G. Laframboise, Phys. Pl. 13, 073503 (2006).

${ }^{35}$ V. N. Duarte and R. A. Clemente, Phys. Pl. 18, 043504 (2011).
${ }^{36}$ M. J. M. Parrot, L. R. O. Storey, L. W. Parker, and J. G. Laframboise, Phys. Fl. 25, 2388 (1982).

${ }^{37}$ P. Cagas, A. Hakim, J. Juno, and B. Srinivasan, Phys. Pl. 24, 022118 (2017).

${ }^{38}$ J. Loizu, P. Ricci, and C. Theiler, Phys. Rev. E 83, 016406 (2011).

${ }^{39}$ S. D. Baalrud and C. C. Hegna, Plasma Sources Sci. Technol. 20, 025013 (2011).

${ }^{40}$ S. D. Baalrud, J. D. Callen, and C. C. Hegna, Phys. Rev. Lett. 102, 245005 (2009).

${ }^{41}$ N. Sternberg and V. Godyak, IEEE Trans. Plasma Sci. 35, 1341 (2007).

${ }^{42}$ L. C. Woods, An Introduction to the Kinetic Theory of Gases and Magnetoplasmas (Oxford University Press Inc., New York, 1993) p. 46.

${ }^{43}$ F. F. Chen and D. Arnush, Phys. Pl. 8, 5051 (2001).

${ }^{44}$ N. Hershkowitz, Phys. Pl. 12, 055502 (2005).

${ }^{45}$ K.-U. Riemann, IEEE Trans. Pl. Sci. 23, 709 (1995).

${ }^{46}$ S. D. Baalrud, B. Scheiner, B. Yee, M. Hopkins, and E. Barnat, Plama Phys. Control. Fusion 57, 044003 (2015).

${ }^{47}$ P. M. Chung, L. Talbot, and K. J. Touryan, Electric Probes in Stationary and Flowing Plasmas: Theory and Application (Springer-Verlag, New York, 1975).

${ }^{48}$ F. F. Chen, J. Appl. Phys. 36, 675 (1965).

${ }^{49}$ F. F. Chen, Plasma Sources Sci. Technol. 18, 035012 (2009).

${ }^{50}$ F. F. Chen, Phys. Pl. 8, 3029 (2001). 
(a)

wall $V_{a}$

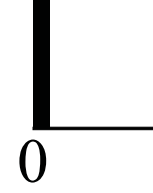

collisionless

plasma $u_{i 0}$ particle flux source, density $n_{0}$ potential $V_{0}$ (b) probe

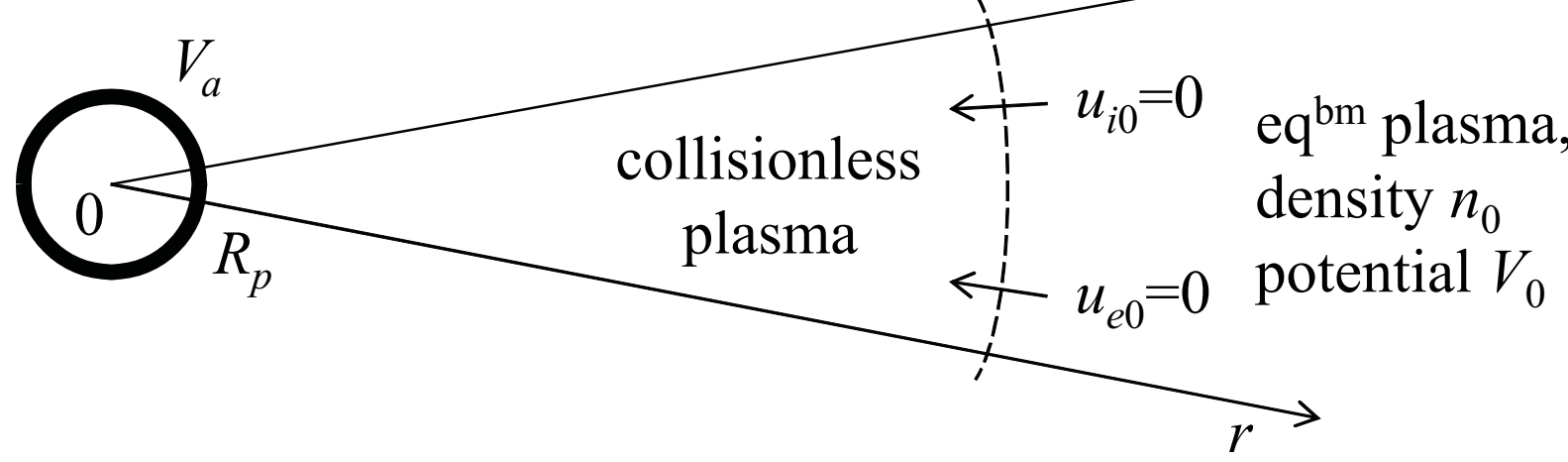

(c)

big probe

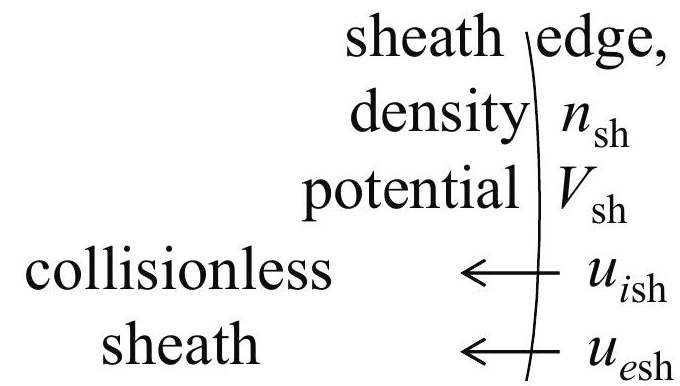

sheath jedge,

potential $V_{\text {sh }}$

quasi-neutral pre-sheath $\mathrm{eq}^{\mathrm{bm}}$ plasma, density $n_{0}$ potential $V_{0}$
$V_{a}$

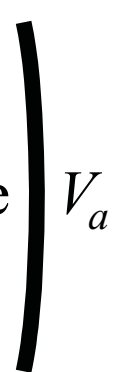




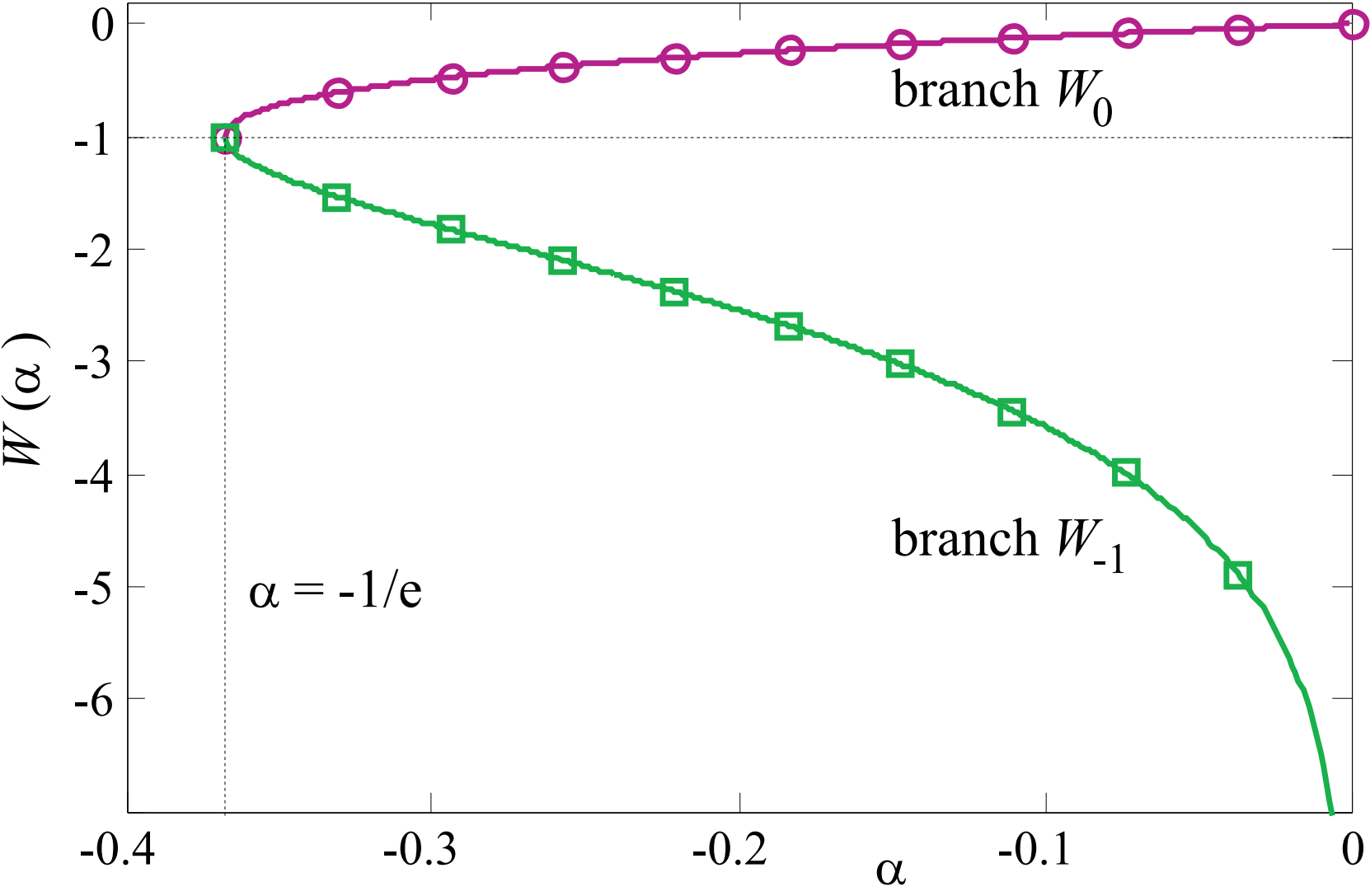




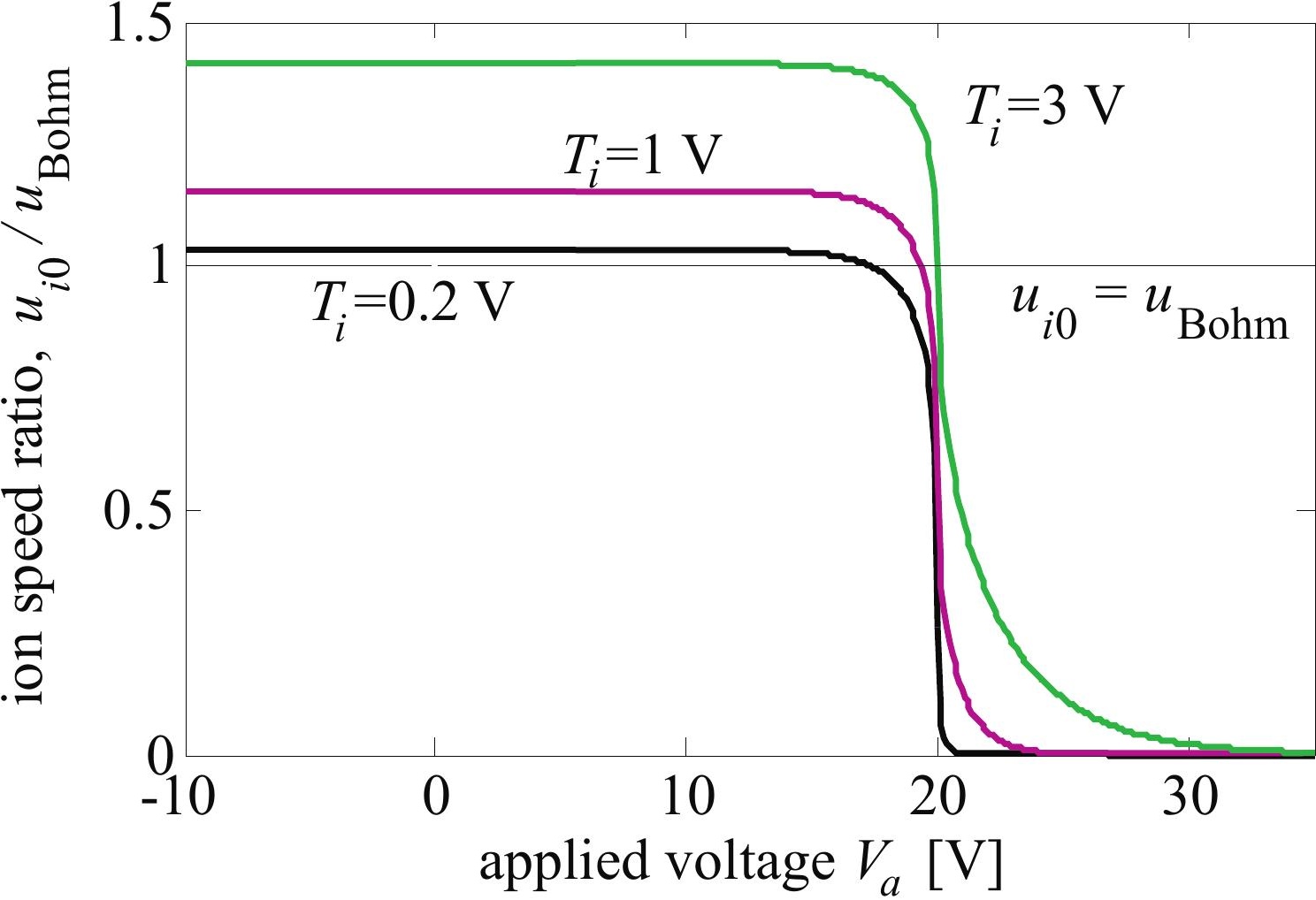




\section{ion velocity at the wall $p$}

ion initial velocity $\sigma U_{i 0}$

e thermal velocity at the wall

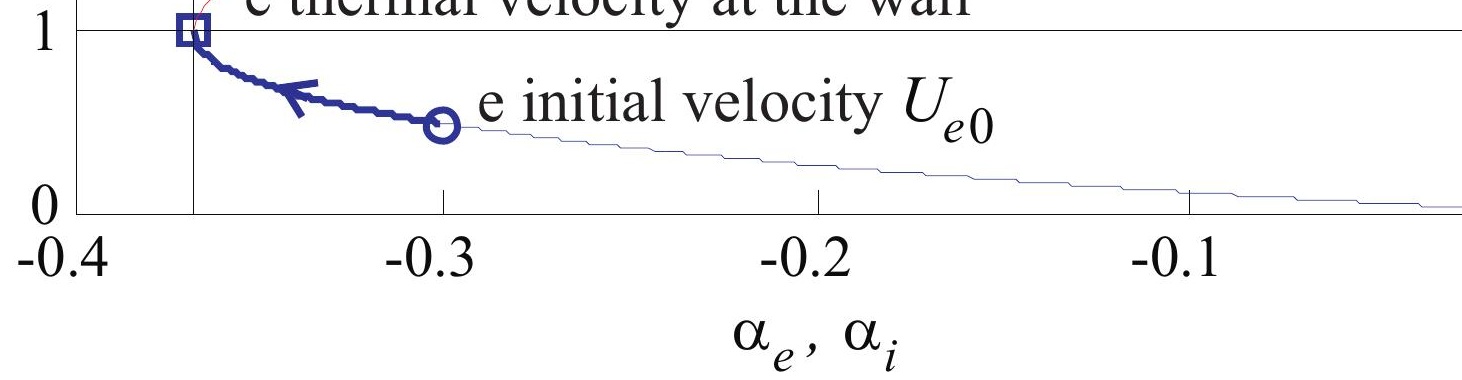




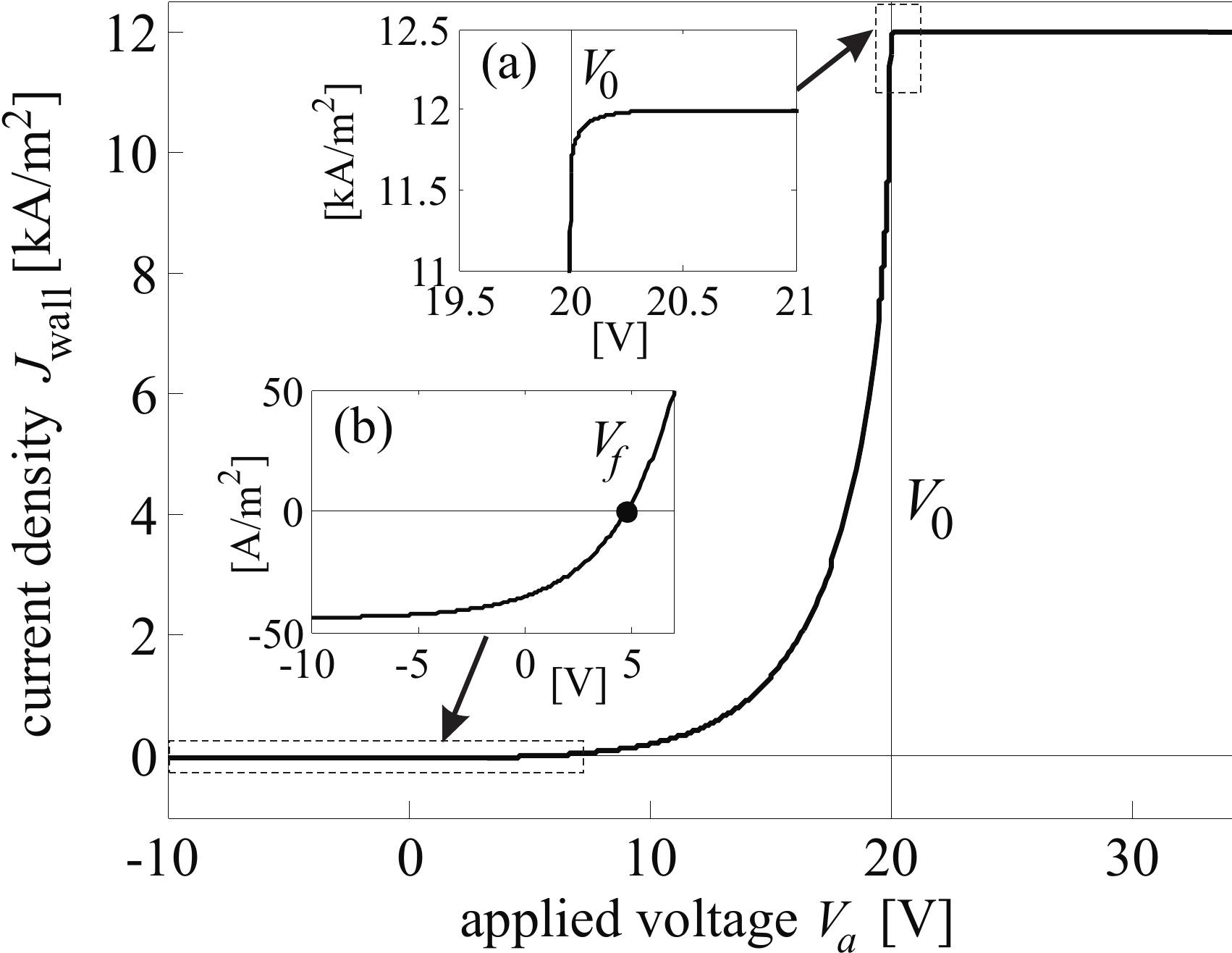




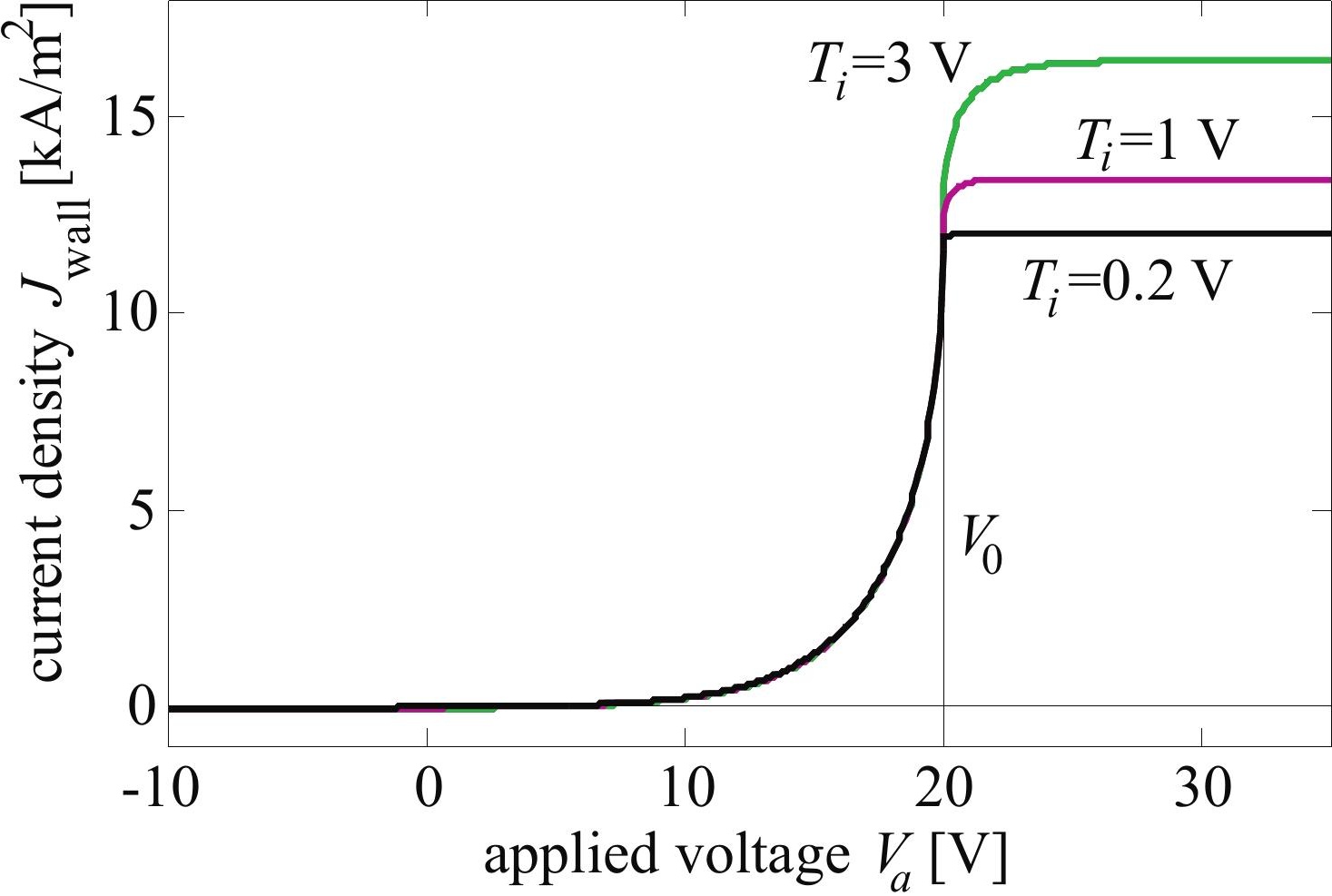



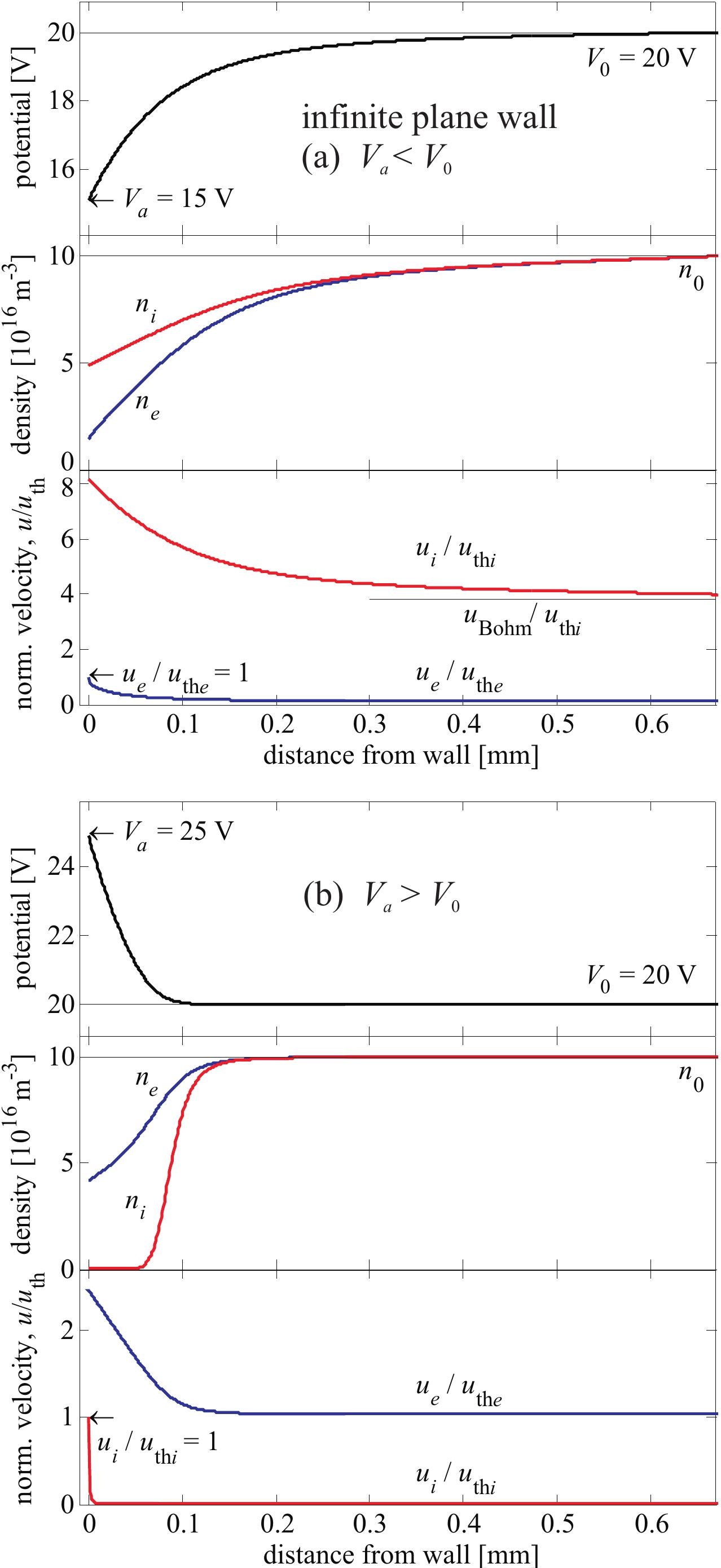


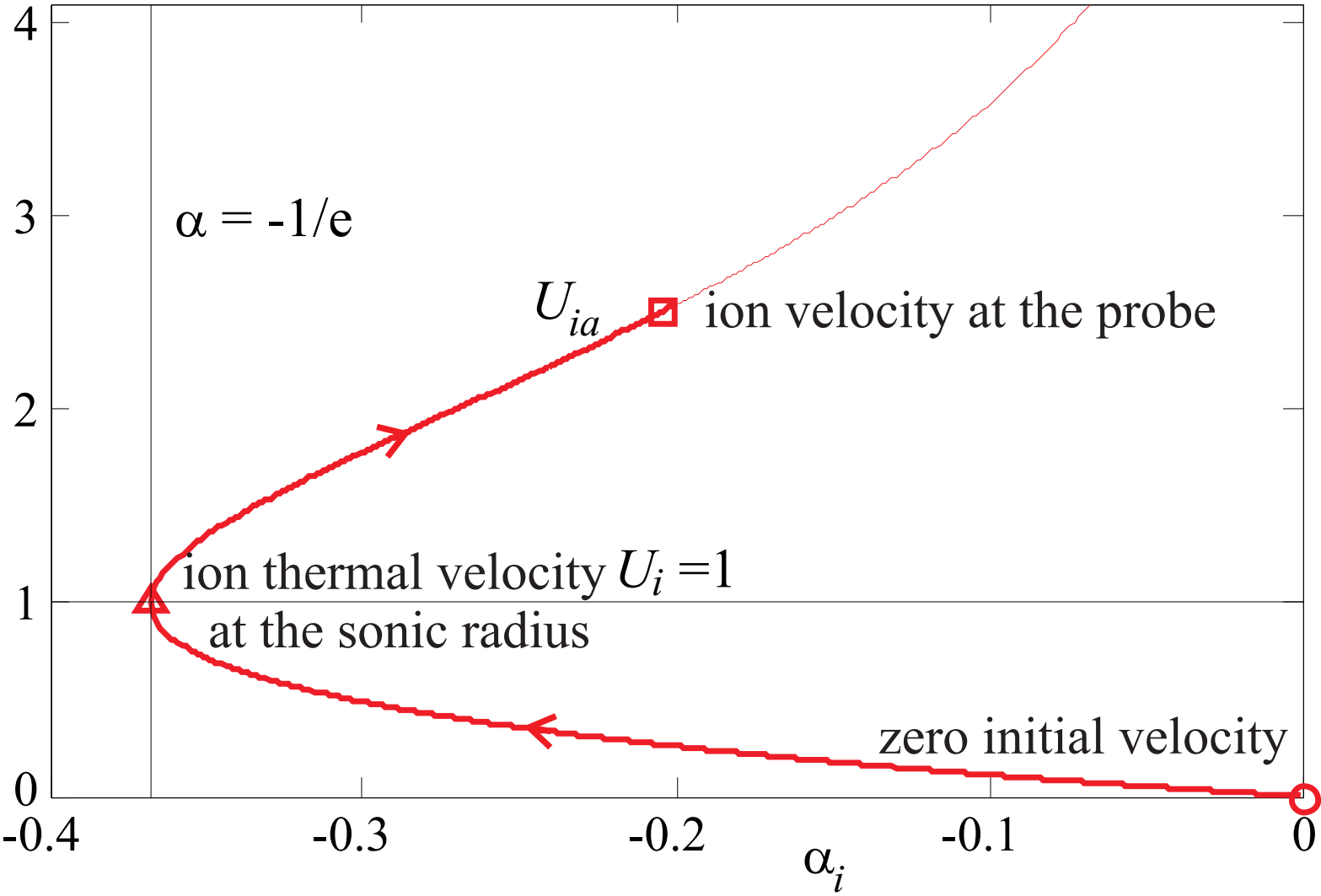




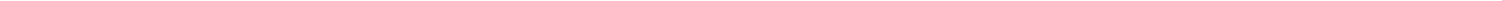




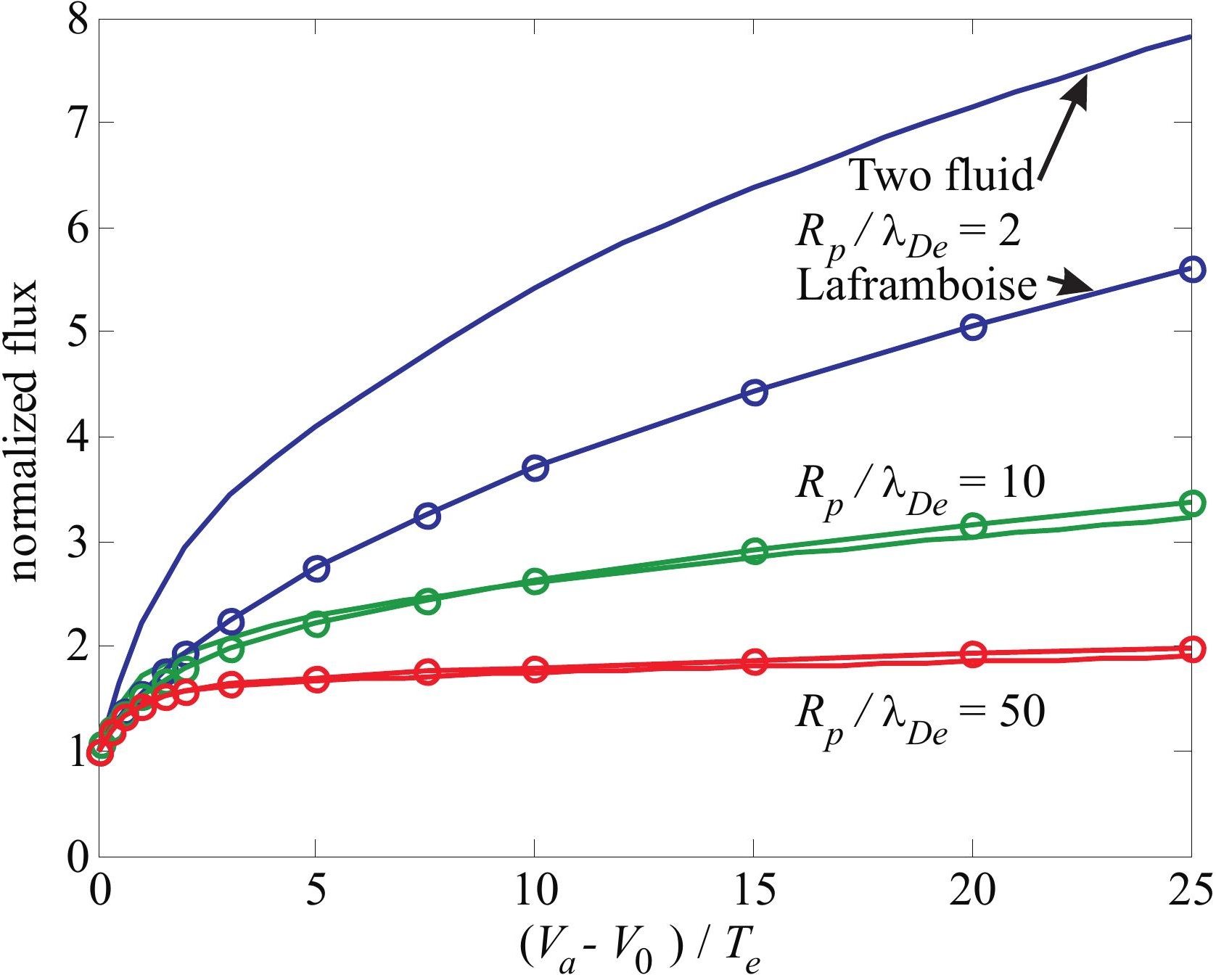




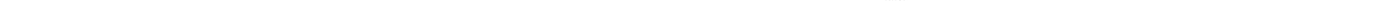




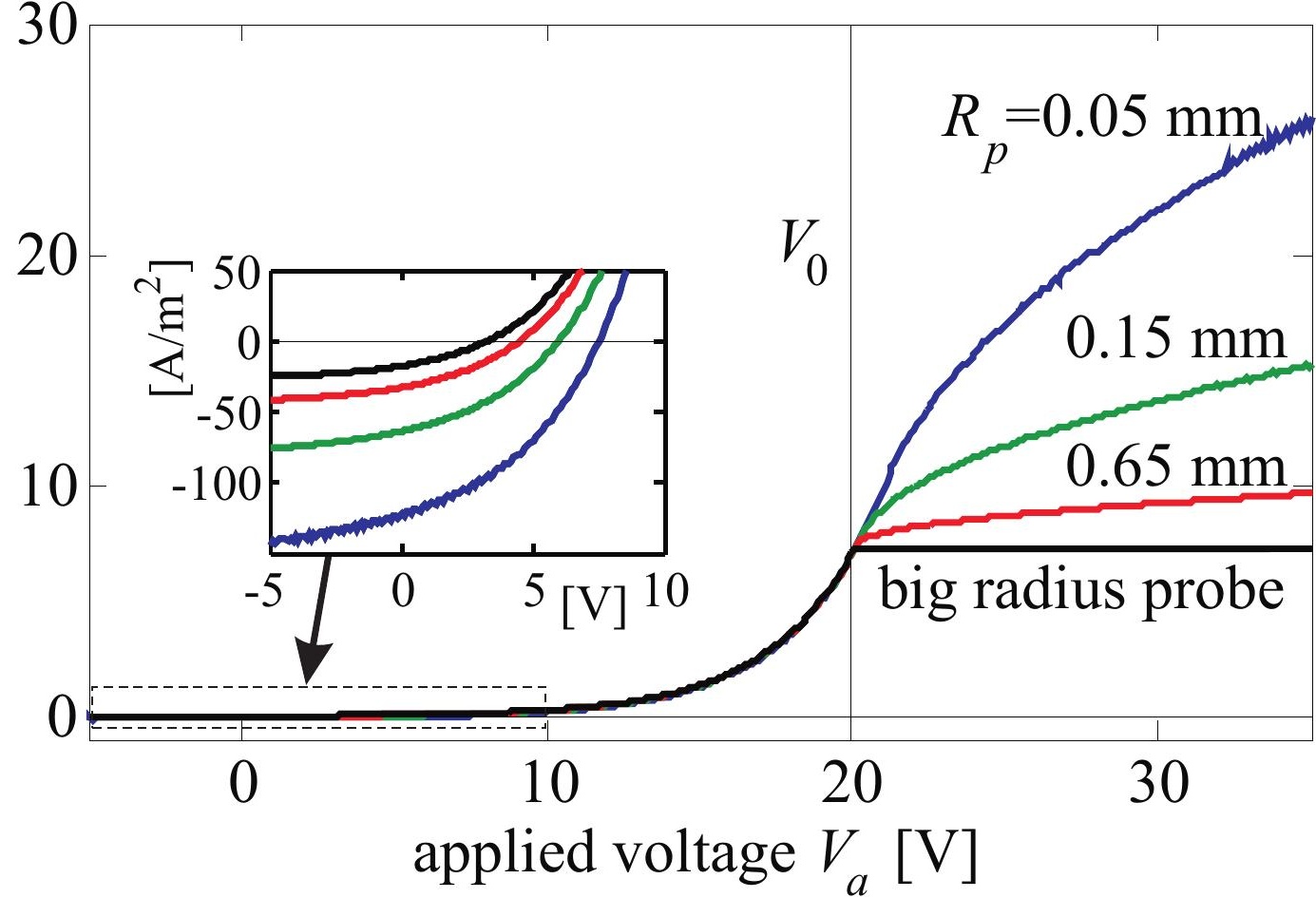



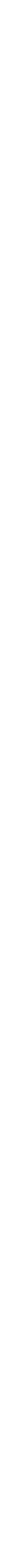


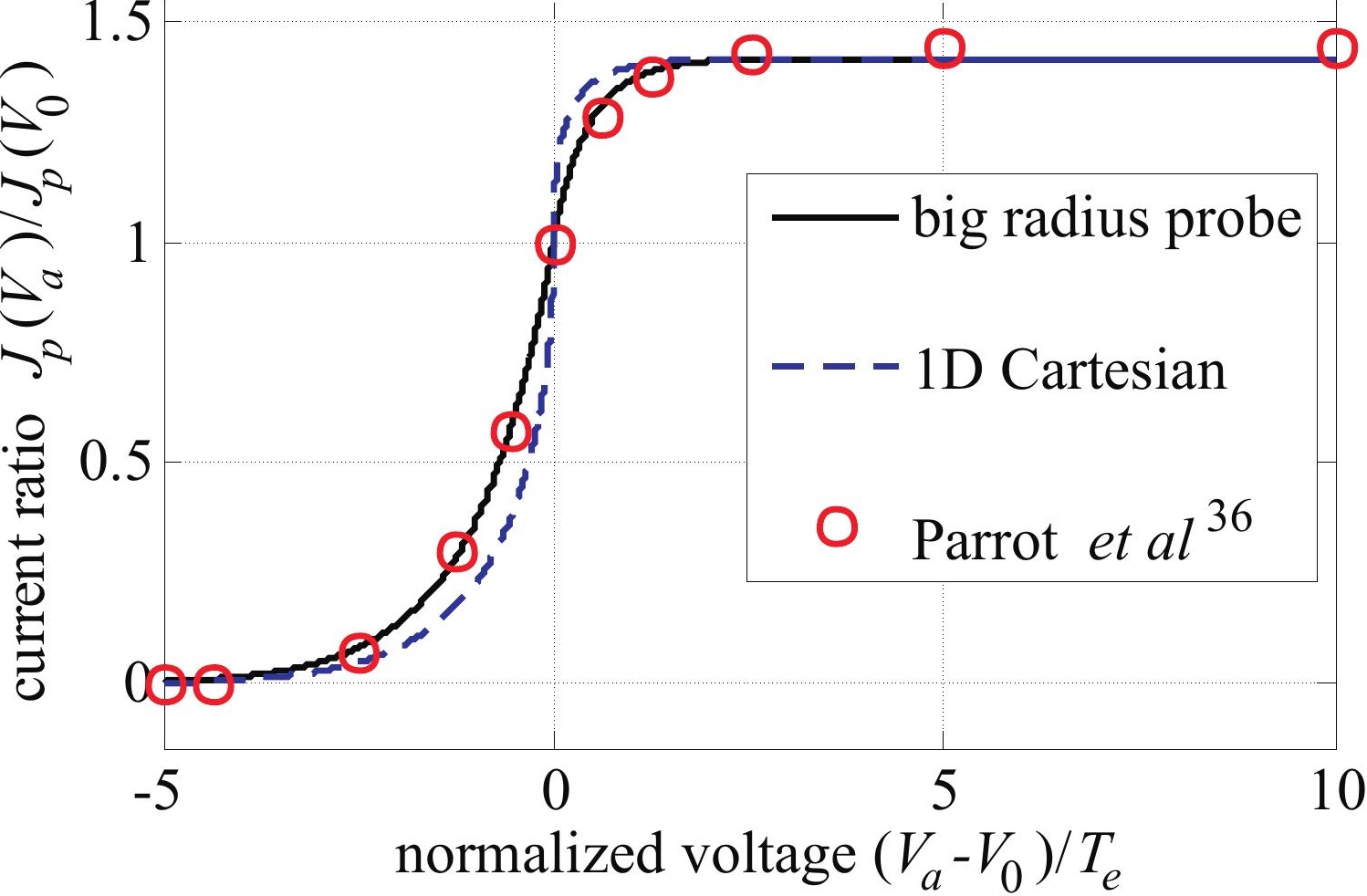




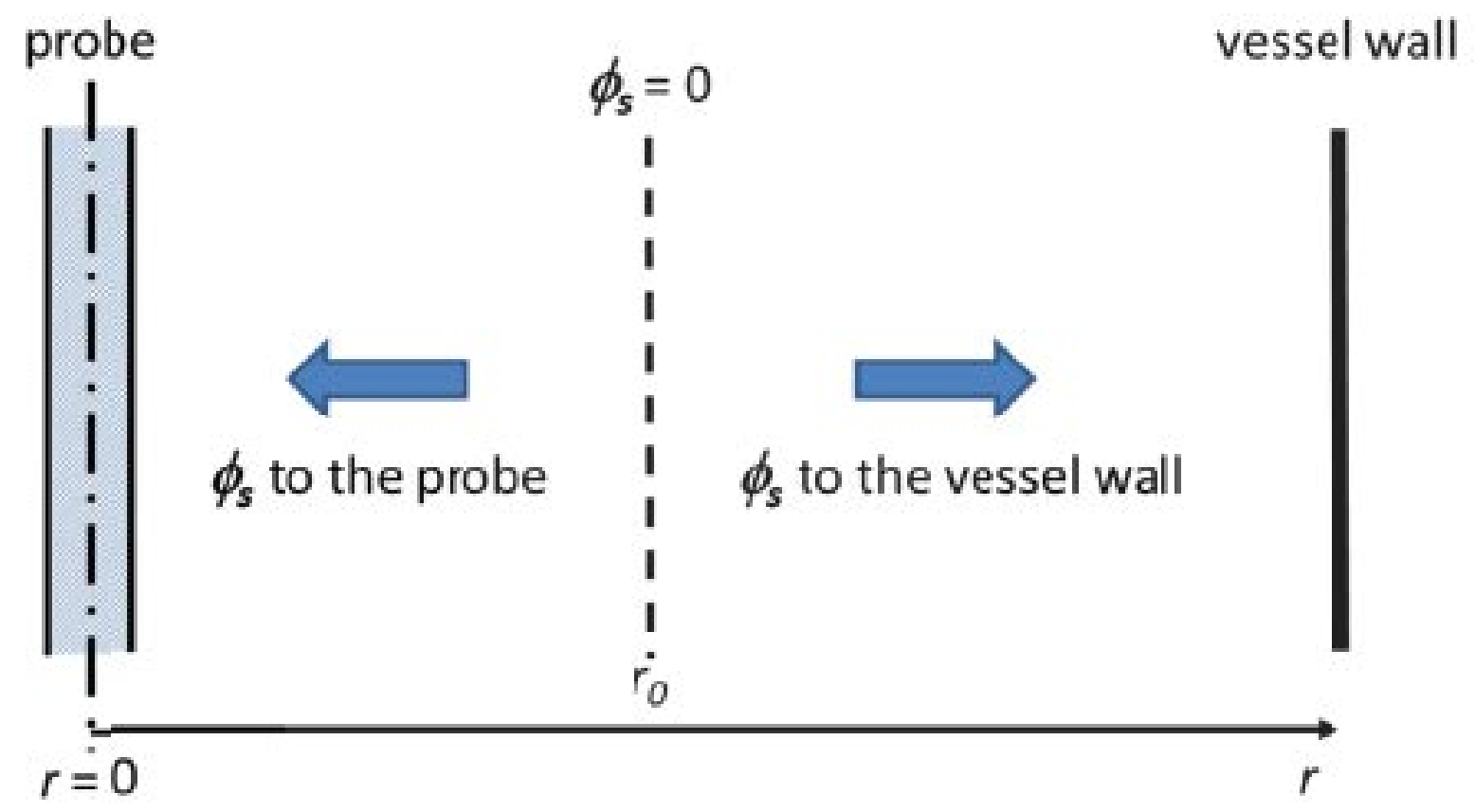




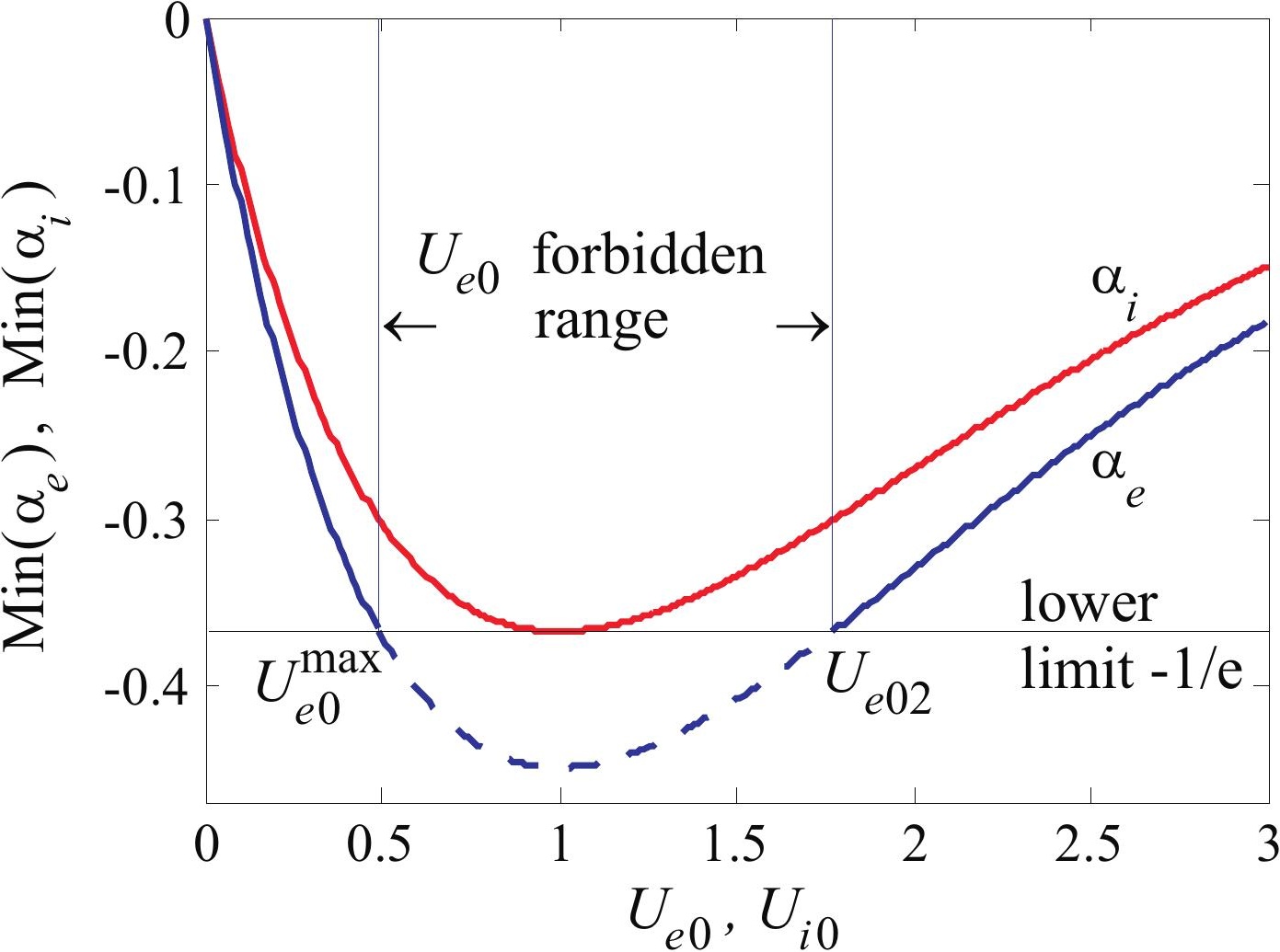



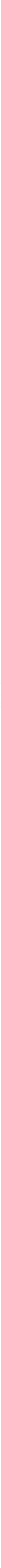


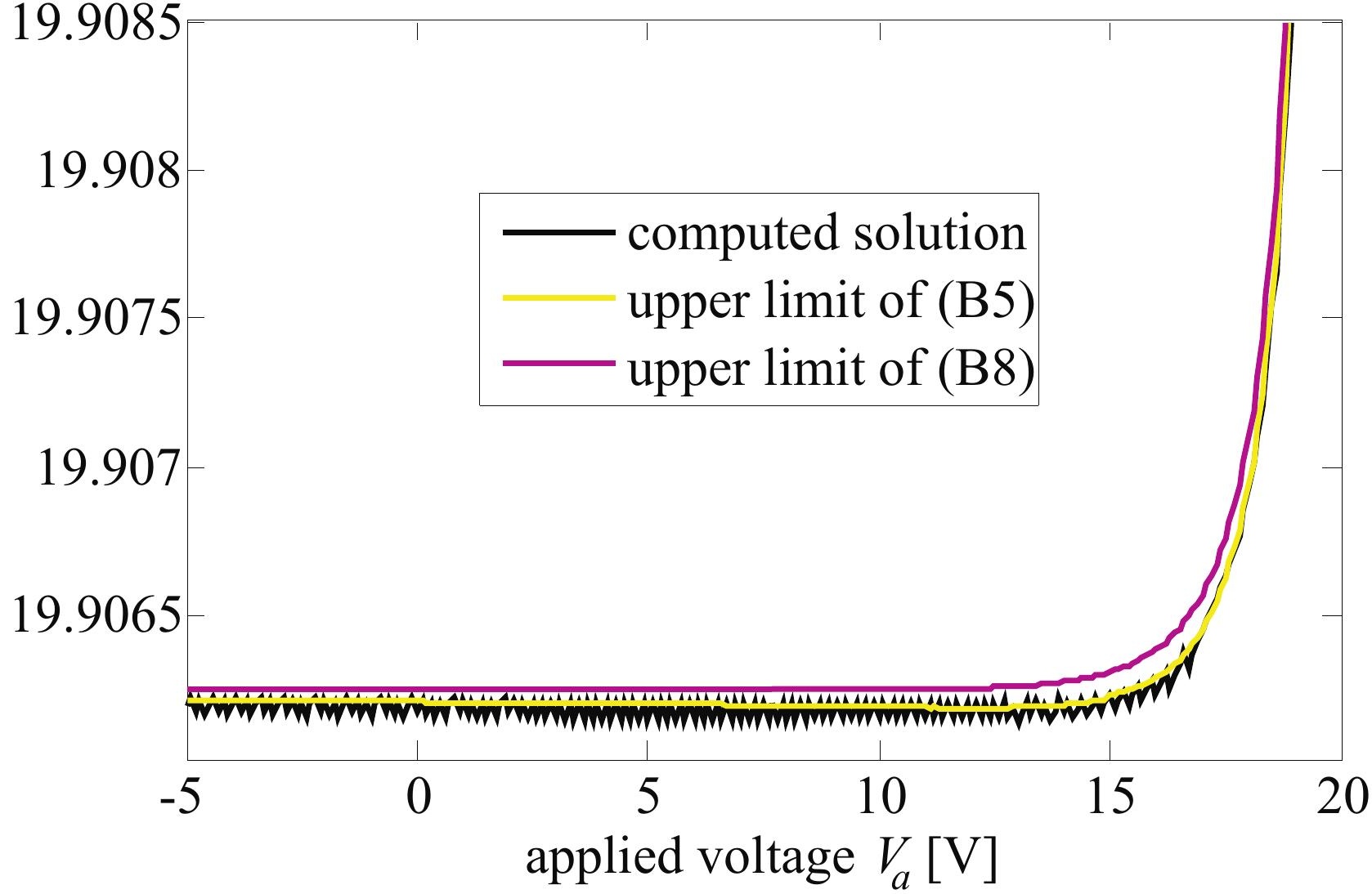



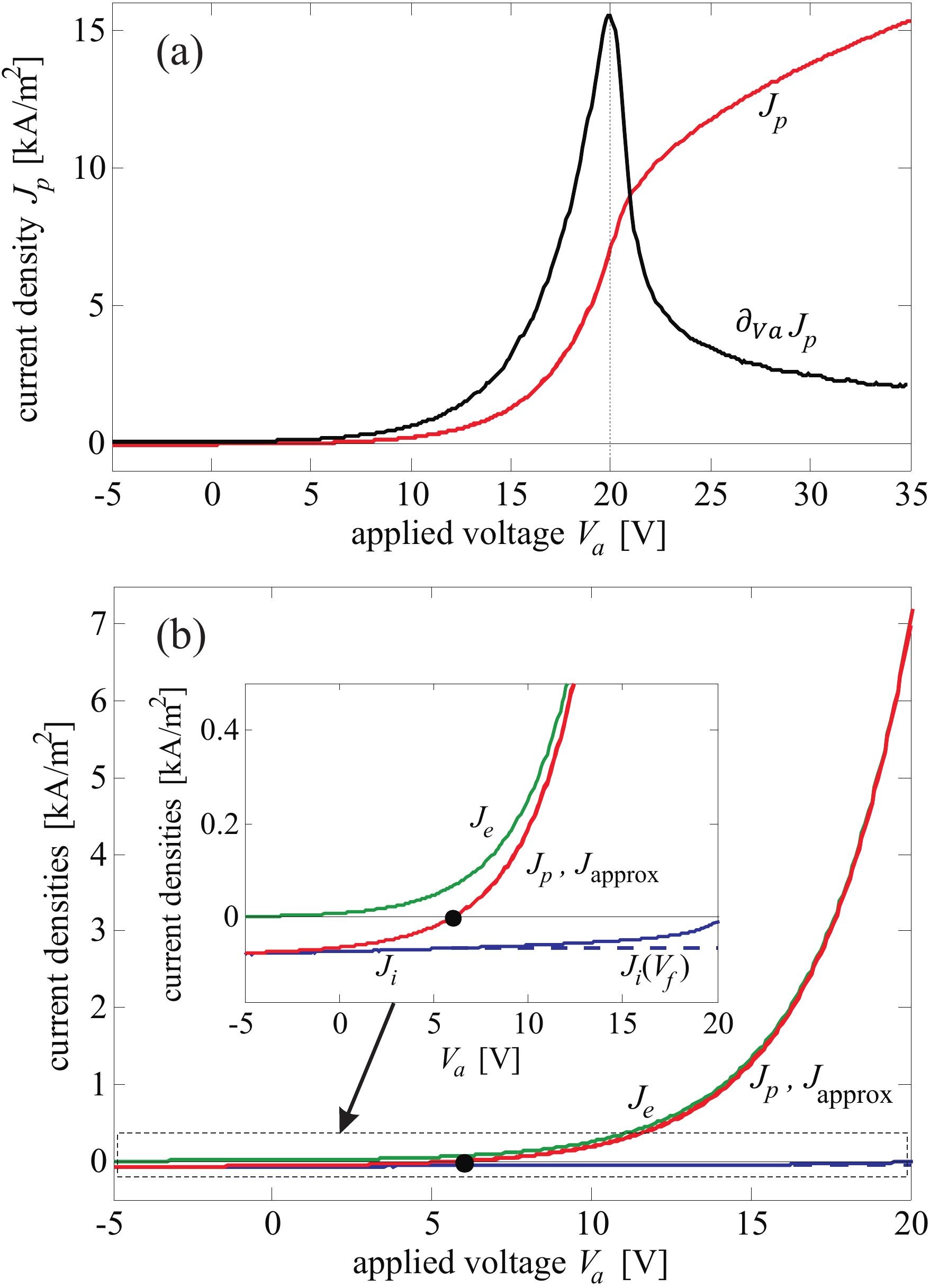